\title{
Transverse beam dynamics in non-linear Fixed Field Alternating Gradient accelerators
}

\author{
M. Haj Tahar, F. Meot
}

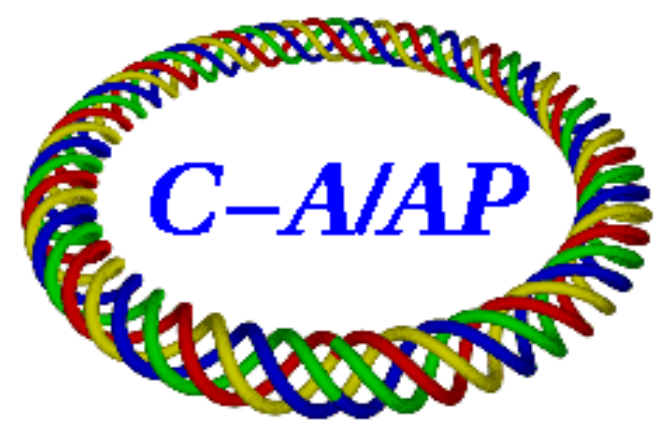

\section{Collider-Accelerator Department Brookhaven National Laboratory Upton, NY 11973}

\author{
U.S. Department of Energy \\ Office of Science, Office of Nuclear Physics
}

Notice: This document has been authorized by employees of Brookhaven Science Associates, LLC under Contract No. DE-SC0012704 with the U.S. Department of Energy. The United States Government retains a nonexclusive, paid-up, irrevocable, world-wide license to publish or reproduce the published form of this document, or allow others to do so, for United States Government purposes. 


\title{
Transverse beam dynamics in non-linear Fixed Field Alternating Gradient accelerators
}

\author{
M. Haj Tahar and F. Méot \\ Brookhaven National Laboratory \\ Upton, NY 11973, USA \\ mhajtahar@bnl.gov
}

\begin{abstract}
In this paper, we present some aspects of the transverse beam dynamics in Fixed Field Ring Accelerators (FFRA): we start from the basic principles in order to derive the linearized transverse particle equations of motion for FFRA, essentially FFAGs and cyclotrons are considered here. This is a simple extension of a previous work valid for linear lattices that we generalized by including the bending terms to ensure its correctness for FFAG lattice. The space charge term (contribution of the internal coulombian forces of the beam) is contained as well, although it is not discussed here. The emphasis is on the scaling FFAG type: a collaboration work is undertaken in view of better understanding the properties of the $150 \mathrm{MeV}$ scaling FFAG at KURRI in Japan, and progress towards high intensity operation. Some results of the benchmarking work between different codes are presented. Analysis of certain type of field imperfections revealed some interesting features about this machine that explain some of the experimental results and generalize the concept of a scaling FFAG to a non-scaling one for which the tune variations obey a well defined law.
\end{abstract}

PACS numbers:

\section{BEAM DYNAMICS IN FFAG IN GENERAL}

In a circular accelerator, the lattice is essentially dominated by several magnets, focusing and defocusing to ensure the bending and/or the focusing of the beam. These magnets are separated by drift space used for insertion devices or accelerating cavities to ensure the basic functions of an accelerator.

\section{Transverse particle equations of motion including bending and space charge terms}

\section{On-momentum transverse equation of motion:}

This is a simple extension of previous work [1]. We first write the equation of motion in a local curvilinear coordinate system $(\mathrm{x}, \mathrm{y}, \mathrm{s})$ following the reference orbit by applying the relativistic form of Newton's second law and including the centrifugal force to account for the rotational movement:

$$
\text { Horizontal restoring force }=\text { Centrifugal force }+ \text { Electromagnetic force }
$$

or in equivalent notation:

$$
\mathbf{F}_{\mathbf{h}}=\mathbf{F}_{\mathbf{c}}+\mathbf{F}_{\mathbf{e m}}
$$

A correct description of the particle motion should invoke the relativity. Although Newton's second law is not valid in the relativistic case, it can be re-written in a covariant relativistic form, which ensures that the equation is valid in all inertial frames [3]. Thus, the Lorentz force is entirely in accord with the special relativity. However, such is not the case in rotating frames which are non-inertial. For a rotating frame, the geometry is non-Euclidean and the general relativity should be invoked [4]. However, we will not do the treatment here. We simply introduce a fictitious force, the centrifugal force, according to Newton's definition, to account for the rotation of the reference frame with respect to the global reference frame. A more elaborate calculation (that avoids the use of the centrifugal force) using the transformation from cartesian to curvilinear coordinate system yields the same result for the linearised particle equations of motion 20].

Now, we calculate each term of the above equation in the transverse plane (x,y) separately, using the path variable $\mathrm{s}$ rather than the time as the independent variable [21].

$$
\begin{aligned}
\mathbf{F}_{\mathbf{h}} & =\frac{d}{d t}\left(m \gamma_{i} \mathbf{v}_{\perp \mathbf{i}}\right)=m v_{z i} \frac{d}{d s}\left(\gamma_{i} v_{z i} \frac{d}{d s} \mathbf{x}_{\perp \mathbf{i}}\right) \\
& =m \gamma_{i} v_{z i}^{2} \frac{d^{2}}{d s^{2}} \mathbf{x}_{\perp \mathbf{i}}+m v_{z i}\left(\frac{d}{d s} \mathbf{x}_{\perp \mathbf{i}}\right) \frac{d}{d s}\left(\gamma_{i} v_{z i}\right) \\
& \simeq m \gamma_{b} \beta_{b}^{2} c^{2} \mathbf{x}_{\perp \mathbf{i}}^{\prime \prime}+m \beta_{b} c^{2}\left(\gamma_{b} \beta_{b}\right)^{\prime} \mathbf{x}_{\perp \mathbf{i}}^{\prime}
\end{aligned}
$$

Thus,

$$
\mathbf{F}_{\mathbf{h}}=m \gamma_{b} \beta_{b}^{2} c^{2}\left[\mathbf{x}_{\perp \mathbf{i}}^{\prime \prime}+\frac{\left(\gamma_{b} \beta_{b}\right)^{\prime}}{\left(\gamma_{b} \beta_{b}\right)} \mathbf{x}_{\perp \mathbf{i}}^{\prime}\right]
$$


It is important to note that $\gamma_{i}$ in Eq. (3) is the relativistic gamma factor defined by:

$$
\gamma_{i}=\frac{1}{\sqrt{1-\beta_{i}^{2}}}=\frac{1}{\sqrt{1-\left(\beta_{i, x}^{2}+\beta_{i, y}^{2}+\beta_{i, z}^{2}\right)}}
$$

However, in the paraxial approximation, the transverse moments of the particle can be neglected compared to its axial moment $\left(\beta_{i, x, y} \ll \beta_{i, z}\right)$ and the bunch mean axial velocity can be defined:

$$
\beta_{b}=\frac{1}{N} \sum_{i=1}^{N} \frac{\mathbf{v}_{\mathbf{i}} \cdot \mathbf{u}_{\mathbf{z}}}{c} \quad ; \quad \mathrm{N}=\text { number of particle per bunch }
$$

Thus, in the derivation above, the particle gamma factor $\gamma_{i}$ was replaced with the bunch gamma factor $\gamma_{b}$. Next, we write:

$$
\mathbf{F}_{\mathbf{c}}=\frac{m \gamma_{i} v_{i}^{2}}{r_{i}} \mathbf{u}_{\mathbf{x}} \simeq \frac{m \gamma_{b} \beta_{b}^{2} c^{2}}{r_{i}} \mathbf{u}_{\mathbf{x}}
$$

Also [1],

$$
\mathbf{F}_{\mathbf{e m}} \simeq q \mathbf{E}_{\perp}^{\mathbf{a}}+q \beta_{b} c \mathbf{z} \times \mathbf{B}_{\perp}^{\mathbf{a}}+q B_{z}^{a} \beta_{b} c \mathbf{x}_{\perp \mathbf{i}}^{\prime} \times \mathbf{z}-q \frac{1}{\gamma_{b}^{2}} \frac{\partial \phi}{\partial \mathbf{x}_{\perp}}
$$

Now, inserting Equations (4), (7) and (8) into (2), one obtains:

$$
\mathbf{x}_{\perp}^{\prime \prime}+\frac{\left(\gamma_{b} \beta_{b}\right)^{\prime}}{\left(\gamma_{b} \beta_{b}\right)} \mathbf{x}_{\perp}^{\prime}=\frac{1}{r_{i}} \mathbf{u}_{\mathbf{x}}+\frac{q}{m \gamma_{b} \beta_{b}^{2} c^{2}} \mathbf{E}_{\perp}^{\mathbf{a}}+\frac{q}{m \gamma_{b} \beta_{b} c} \mathbf{z} \times \mathbf{B}_{\perp}^{\mathbf{a}}+\frac{q B_{z}^{a}}{m \gamma_{b} \beta_{b} c} \mathbf{x}_{\perp}^{\prime} \times \mathbf{z}-\frac{q}{m \gamma_{b}^{3} \beta_{b}^{2} c^{2}} \frac{\partial \phi}{\partial \mathbf{x}_{\perp}}
$$

which is the general particle equation of motion in the transverse plane.

-Next we write, $r_{i}=\rho+x$, where $\rho$ is the bending radius of the equilibrium orbit. Using a Taylor expansion to the first order, this gives:

$$
\frac{1}{r_{i}}=\frac{1}{\rho+x}=\frac{1}{\rho}\left(1+\frac{x}{\rho}\right)^{-1} \simeq \frac{1}{\rho}\left(1-\frac{x}{\rho}\right)=\frac{1}{\rho}-\frac{x}{\rho^{2}}
$$

Substituting Eq (10) into (9), and projecting onto the horizontal plane (x), one obtains:

$$
x^{\prime \prime}+\frac{\left(\gamma_{b} \beta_{b}\right)^{\prime}}{\left(\gamma_{b} \beta_{b}\right)} x^{\prime}+\frac{1}{\rho^{2}} x=\frac{1}{\rho}+\frac{q}{m \gamma_{b} \beta_{b}^{2} c^{2}} E_{x}^{a}-\frac{q}{m \gamma_{b} \beta_{b} c} B_{y}^{a}+\frac{q B_{z}^{a}}{m \gamma_{b} \beta_{b} c} y^{\prime}-\frac{q}{m \gamma_{b}^{3} \beta_{b}^{2} c^{2}} \frac{\partial \phi}{\partial x}
$$

The Taylor expansion of the field around the median plane yields:

$$
\begin{aligned}
B_{y}^{a} & \simeq B_{y 0}^{a}+\frac{\partial B_{y}^{a}}{\partial x} x=B_{y 0}^{a}\left(1+\frac{\rho}{B_{y 0}^{a}} \frac{\partial B_{y}^{a}}{\partial x} \frac{x}{\rho}\right)=B_{y 0}^{a}\left(1-n \frac{x}{\rho}\right) \\
& =\frac{m \gamma_{b} \beta_{b} c}{q} \frac{1}{\rho}\left(1-n \frac{x}{\rho}\right)
\end{aligned}
$$

where $n=-\frac{\rho}{B_{y 0}^{a}} \frac{\partial B_{y}^{a}}{\partial x}$ is the field index.

Next, we substitute Eq (12) into Eq (11), the x-transverse equation of motion becomes:

$$
x^{\prime \prime}+\frac{\left(\gamma_{b} \beta_{b}\right)^{\prime}}{\left(\gamma_{b} \beta_{b}\right)} x^{\prime}+\frac{1-n}{\rho^{2}} x=\frac{q}{m \gamma_{b} \beta_{b}^{2} c^{2}} E_{x}^{a}+\frac{q B_{z}^{a}}{m \gamma_{b} \beta_{b} c} y^{\prime}-\frac{q}{m \gamma_{b}^{3} \beta_{b}^{2} c^{2}} \frac{\partial \phi}{\partial x}
$$

It shows that any longitudinal component of the magnetic field introduces coupling.

-Now, projecting Eq (9) onto the vertical plane yields:

$$
y^{\prime \prime}+\frac{\left(\gamma_{b} \beta_{b}\right)^{\prime}}{\left(\gamma_{b} \beta_{b}\right)} y^{\prime}=\frac{q}{m \gamma_{b} \beta_{b}^{2} c^{2}} E_{y}^{a}+\frac{q}{m \gamma_{b} \beta_{b} c} B_{x}^{a}-\frac{q B_{z}^{a}}{m \gamma_{b} \beta_{b} c} x^{\prime}-\frac{q}{m \gamma_{b}^{3} \beta_{b}^{2} c^{2}} \frac{\partial \phi}{\partial y}
$$

Also, in the vacuum aperture of the accelerator, the Maxwell equations must satisfy:

$$
\left\{\begin{array}{c}
\nabla \cdot \mathbf{B}^{a}=0 \\
\nabla \times \mathbf{B}^{a}=0
\end{array}\right.
$$


which yields

$$
\frac{\partial B_{x}^{a}}{\partial y}-\frac{\partial B_{y}^{a}}{\partial x}=0 \Longrightarrow B_{x}^{a}=-\frac{m \gamma_{b} \beta_{b} c}{q} \frac{n}{\rho^{2}} y
$$

so that the $\mathrm{y}$-transverse particle equation of motion becomes:

$$
y^{\prime \prime}+\frac{\left(\gamma_{b} \beta_{b}\right)^{\prime}}{\left(\gamma_{b} \beta_{b}\right)} y^{\prime}+\frac{n}{\rho^{2}} y=\frac{q}{m \gamma_{b} \beta_{b}^{2} c^{2}} E_{y}^{a}-\frac{q B_{z}^{a}}{m \gamma_{b} \beta_{b} c} x^{\prime}-\frac{q}{m \gamma_{b}^{3} \beta_{b}^{2} c^{2}} \frac{\partial \phi}{\partial y}
$$

NB: The beam potential $\phi$ generated from the charge distribution will be treated in detail when the problem of the space charge forces is tackled. For the moment one shall bear in mind that, for a single particle theory, the contribution of the last term in the transverse equations of motion can be neglected.

\section{Off-momentum equation of motion:}

The preceding analysis applies to particles having the same momenta. Here we investigate the effect of a momentum change $\Delta p$ : We assume that the beam is an ensemble of $\mathrm{N}$ particles having different momenta, within a certain interval called momentum spread of the beam $\Delta p / p$. This is equivalent to replacing $\beta \gamma m c=p$ by $p+\Delta p$ in the preceding equation.

Starting from Eq (11), one obtains:

$$
\begin{aligned}
x^{\prime \prime}+\frac{\left(\gamma_{b} \beta_{b}\right)^{\prime}}{\left(\gamma_{b} \beta_{b}\right)} x^{\prime}+\frac{1-n}{\rho^{2}} x= & \frac{\delta}{\rho} \\
& +\frac{q}{m \gamma_{b} \beta_{b}^{2} c^{2}} \frac{1}{1+\delta} E_{x}^{a} \\
& +\frac{q B_{z}^{a}}{m \gamma_{b} \beta_{b} c} \frac{1}{1+\delta} y^{\prime}-\frac{q}{m \gamma_{b}^{3} \beta_{b}^{2} c^{2}} \frac{1}{(1+\delta)^{2}} \frac{\partial \phi}{\partial x}
\end{aligned}
$$

where $\delta=\Delta p / p$ and $1 / \rho=q B_{y}^{a} / p$. Solving this equation yields a solution of the homogeneous equation plus a particular solution of the inhomogeneous equation. The actual particle coordinate is thus a superposition of the betatron oscillation plus an additional contribution due to momentum errors. The off-momentum solution can be found by using the variation of parameters method.

\section{On solving the transverse equations of motion:}

In the accelerator physics literature, it is generally assumed that, when the damping term is removed, the above equations are of the form of a Hill's equation, for which general solutions exist and can be written in a closed form, using Floquet theory. The main assumption that allows to obtain such a result is that the coefficients of the above equation are periodic in time (or s). This gives rise to an amplitude function $A(s)=\sqrt{\beta(s)}$ that varies periodically as well. In reality, this may be a good approximation in certain cases, for instance a synchrotron where the accelerated orbit coincides with the closed orbit, but it may not be a good approximation for other types of circular machines such as FFAGs where the accelerated orbit moves outwards and therefore the coefficients of the above equation are no longer periodic in s. In what follows, we use the method of Wentzel-Kramers-Brillouin (WKB) in order to find approximate solutions of the equation of motion.

\section{The WKB approximation:}

The previous equations of motion (Eq13 \& Eq[17), when there is no applied transverse electric field neither a longitudinal magnetic field component, and neglecting the space charge term become:

$$
\left\{\begin{array}{c}
x^{\prime \prime}+\frac{\left(\gamma_{b} \beta_{b}\right)^{\prime}}{\left(\gamma_{b} \beta_{b}\right)} x^{\prime}+\frac{1-n}{\rho^{2}} x=0 \\
y^{\prime \prime}+\frac{\left(\gamma_{b} \beta_{b}\right)^{\prime}}{\left(\gamma_{b} \beta_{b}\right)} y^{\prime}+\frac{n}{\rho^{2}} y=0
\end{array}\right.
$$

The above equations can be written in the standard form:

$$
x^{\prime \prime}+p(s) x^{\prime}+q(s) x=0
$$

This is a 2nd order linear differential equation with variable coefficients, for which, in general, a closed form solution is not known. We will first seek to remove the first term derivative in the above equation in order to simplify the analysis. 
In what follows, we use a method that has been developed in [5] in order to express approximate analytical solutions of the point-reactor differential equations when the reactivity is an arbitrary specified function of time:

Without any loss of generality, we assume that a solution of Eq (20) can be written in the form: $x(s)=u(s) v(s)$. Thus Eq (20) becomes:

$$
u v^{\prime \prime}+\left(2 u^{\prime}+p u\right) v^{\prime}+\left(u^{\prime \prime}+p u^{\prime}+q u\right) v=0
$$

and one only has to choose the function $u(s)$ such that $2 u^{\prime}+p u=0$, i.e,

$$
u(s)=\exp \left(-\frac{1}{2} \int_{s_{0}}^{s} p(h) d h\right)
$$

Now, the first term derivative is removed by making the Liouville-Green transformation $x(s)=v(s) \cdot \exp \left(-\frac{1}{2} \int_{s_{0}}^{s} p(h) d h\right)$, which yields:

$$
\begin{array}{r}
\frac{d x}{d s}=\left(v^{\prime}-\frac{p}{2} v\right) \exp \left(-\frac{1}{2} \int_{s_{0}}^{s} p(h) d h\right) \\
\frac{d^{2} x}{d s^{2}}=\left(v^{\prime \prime}-\frac{p^{\prime}}{2} v-p v^{\prime}+\frac{p^{2}}{4} v\right) \exp \left(-\frac{1}{2} \int_{s_{0}}^{s} p(h) d h\right)
\end{array}
$$

And by injecting Eq (23) and (24) into (20), one obtains:

$$
v^{\prime \prime}+\left(q(s)-\frac{1}{2} \frac{d p}{d s}-\frac{p^{2}}{4}\right) v=0
$$

Now, back to Eq (19), the above formalism applies by simply replacing $p(s)=\frac{\left(\gamma_{b} \beta_{b}\right)^{\prime}}{\left(\gamma_{b} \beta_{b}\right)}$ which yields:

$$
\begin{aligned}
x(s) & =v(s) \cdot \exp \left(-\frac{1}{2} \int_{s_{0}}^{s} p(h) d h\right)=v(s) \cdot \exp \left(-\frac{1}{2} \int_{s_{0}}^{s} \frac{\left(\gamma_{b} \beta_{b}\right)^{\prime}}{\left(\gamma_{b} \beta_{b}\right)} d h\right) \\
& =\frac{C}{\sqrt{\gamma_{b} \beta_{b}}} v(s)
\end{aligned}
$$

and $v(s)$ is a solution of $\mathrm{Eq}(25)$ above. The above treatment shows why, in general, we refer to the first term derivative in Eq (19) as the "damping term".

Next, we rewrite Eq (25) as:

$$
v^{\prime \prime}-h^{2}(s) v=0 \quad ; \quad h^{2}(s) \in \mathbb{R}_{ \pm}
$$

where

$$
\begin{array}{ll}
h^{2}(s)=-\frac{1-n}{\rho^{2}}+\frac{1}{2} \frac{d}{d s}\left[\frac{\left(\gamma_{b} \beta_{b}\right)^{\prime}}{\left(\gamma_{b} \beta_{b}\right)}\right]+\frac{1}{4}\left[\frac{\left(\gamma_{b} \beta_{b}\right)^{\prime}}{\left(\gamma_{b} \beta_{b}\right)}\right]^{2} \quad ; \quad \text { horizontal plane } \\
h^{2}(s)=-\frac{n}{\rho^{2}}+\frac{1}{2} \frac{d}{d s}\left[\frac{\left(\gamma_{b} \beta_{b}\right)^{\prime}}{\left(\gamma_{b} \beta_{b}\right)}\right]+\frac{1}{4}\left[\frac{\left(\gamma_{b} \beta_{b}\right)^{\prime}}{\left(\gamma_{b} \beta_{b}\right)}\right]^{2} \quad ; \quad \text { vertical plane }
\end{array}
$$

Next, we will seek an approximate solution of Eq (27).

We postulate: $v=\exp [\phi(s)]$ where $\phi(s) \in \mathbb{C}$. Substitution into Eq (27) yields a second order non-linear differential equation:

$$
\frac{d^{2} \phi}{d s^{2}}+\left(\frac{d \phi}{d s}\right)^{2}-h(s)^{2}=0
$$

If $\frac{d \phi}{d s}$ is slowly changing (adiabatic change), an iterative solution can be found. The first iteration is:

$$
\frac{d \phi}{d s} \simeq h(s)
$$

The second iteration is:

$$
\left(\frac{d \phi}{d s}\right)^{2}=h^{2}-\frac{d^{2} \phi}{d s^{2}} \simeq h^{2}-\frac{d h}{d s}
$$


Now, we treat $\frac{d h}{d s}$ as small and we make the distinction between two cases (focusing/defocusing), according to the sign of $h^{2}(s)$ :

$\underline{\text { If } h^{2}(s)>0:}$

$$
\begin{gathered}
\frac{d \phi}{d s} \simeq\left(h^{2}-\frac{d h}{d s}\right)^{1 / 2}=h\left(1-\frac{1}{h^{2}} \frac{d h}{d s}\right)^{1 / 2} \simeq h-\frac{1}{2 h} \frac{d h}{d s} \\
\Longrightarrow \phi \simeq \int h d s-\frac{1}{2} \ln |h| \\
v=\exp [\phi(s)] \simeq \frac{1}{\sqrt{|h|}} \exp \left(\int h d s\right)
\end{gathered}
$$

and a complete approximate solution of Eq. (19) is given by:

$$
x(s)=\frac{1}{\sqrt{|h|}} \frac{1}{\sqrt{\beta_{b} \gamma_{b}}}\left[A \exp \left(\int h d s\right)+B \exp \left(-\int h d s\right)\right]
$$

where $\mathrm{A}$ and $\mathrm{B}$ are constants and $\mathrm{h}$ is defined in $\mathrm{Eq}(28)$ above.

$$
\underline{\text { If } h^{2}(s)<0:}
$$

Repeating the same steps with h imaginary leads to:

$$
x(s)=\frac{1}{\sqrt{|h|}} \frac{1}{\sqrt{\beta_{b} \gamma_{b}}}\left[C \exp \left(i \int|h| d s\right)+D \exp \left(-i \int|h| d s\right)\right]
$$

- At the classical turning point, i.e when $\mathrm{h}=0$, the amplitude function diverges and the above solution is no longer valid. Such a condition is fulfilled at the point where the magnetic field changes its sign, i.e when the gradient is alternated. However, this difficulty can be handled by finding the higher order terms of the WKB approximation.

- The main approximation that we made in this analysis was to assume that the phase $\phi(s)$ was slowly changing which implies that $h^{2}(s)$ defined in Eq. (28) above is slowly changing as well. This adiabatic change becomes less and less valid when the energy gain per turn becomes important, since then, the change in $\gamma_{b} \beta_{b}$ becomes important. This is generally a requirement to limit the space charge effects and also for fast crossing of the resonances.

- The amplitude of the oscillations as well as its frequencies is strongly dependent on the form of the magnetic field (to the first order in this analysis, the bending radius and the field see Eq (28)). This is to remind that any defect in the design of the magnets may be a major source of imperfections and needs to be corrected.

In conclusion, this shows that, for slowly varying parameters of the linearized particle equations of motion, the damping law is given by [22]

$$
\epsilon_{n o r m, r m s}=\beta_{b} \gamma_{b}|h| \times \epsilon_{r m s}
$$

where $\epsilon_{r m s}$ is the rms beam emittance.

This result can also be understood by means of the Poincare-Cartan integral invariant that we will discuss later.

Finally, let's remind the Lagrange formula for the integration of the second order inhomogeneous linear differential equation:

$$
x^{\prime \prime}+p(s) x^{\prime}+q(s) x=f(s)
$$

Knowing a fundamental system of solutions $x_{1}, x_{2}$ of the homogeneous Eq. (20), one can obtain a solution of (38) by means of the formula [12]:

$$
x_{p}(s)=\int_{s_{0}}^{s} \frac{x_{1}(s) x_{2}(s)-x_{2}(s) x_{1}(s)}{W(s)} f(s) d s
$$

where $\mathrm{W}(\mathrm{s})$ is the wronskian of the two fundamental solutions defined by,

$$
W(s)=x_{1}(s) x_{2}^{\prime}(s)-x_{2}(s) x_{1}^{\prime}(s)
$$




\section{BEAM DYNAMICS IN SCALING FFAG}

All of the above remains valid for scaling FFAG. The only difference is, now, we assume that the magnetic field obeys a well defined law, that we generally refer to as the scaling law. The scaling law guarantees that the number of betatron oscillations remain constant [8]. However, it is susceptible to change due to imperfections. Therefore, in our analysis, we decide to take the magnetic field imperfections into account. In this section, we first show the origin of the scaling law for this type of accelerators. Then we summarize some of the benchmarking work undertaken to validate some of the simulation results. We also discuss about the main assumptions for the calculation of the scaling factor from the field map. The emphasis is made on the mathematical correctness of the calculation. We conclude this section by investigating the effect of field imperfections on the transverse beam dynamics of FFAGs.

We first re-write Eq (19) by dropping the damping term and making the transformation $s=\theta R$ where $\theta$ is the azimuthal angle with the periodicity of the machine i.e $2 \pi / N$, N being the number of sectors, and $\mathrm{R}$ is the equivalent radius for each closed orbit, i.e $R=S / 2 \pi$. This is the same transformation that can be found in the original paper by Symon in 1956 [8]. Thus, we obtain:

$$
\left\{\begin{array}{l}
\frac{d^{2} x}{d \theta^{2}}+\left(\frac{R^{2}}{\rho^{2}(R, \theta)}[1-n]\right) x=0 \\
\frac{d^{2} y}{d \theta^{2}}+\left(\frac{R^{2}}{\rho^{2}(R, \theta)} n\right) y=0
\end{array}\right.
$$

Therefore, two sufficient conditions to have both the vertical and horizontal betatron oscillations constant with respect to the momentum are:

$$
\begin{array}{r}
\left.\frac{\partial n}{\partial p}\right|_{\theta=\text { const }}=0 \\
\left.\frac{\partial}{\partial p}\left(\frac{R}{\rho}\right)\right|_{\theta=\text { const }}=0
\end{array}
$$

These conditions are called the cardinal conditions of a scaling FFAG [9]: the first condition expresses the constancy of the field index with respect to the momentum while the second condition expresses the similarity between the different closed orbits. In other words, closed orbits corresponding to different energies are homothetic with respect to the centre of the machine, therefore explaining the scaling property of this type of accelerator. However, one fundamental hidden assumption in this analysis is that the number of betatron oscillations of the accelerated orbit is the same as that obtained from the closed orbits. As was shown in Eq. (27), the forcing term of the equation of motion of the accelerated orbit differs from that of the closed orbits due to the presence of the terms containing the change in momentum of the particles, namely $\left(p^{\prime} / p\right)^{\prime}$ and $\left(p^{\prime} / p\right)^{2}$. However, for small acceleration rates, this effect can be neglected.

More detailed analysis of these properties will be tackled when developing the hard edge model. It results from both cardinal conditions that the field index averaged over the azimuth of the closed orbits is constant as well (independent of the radius). This is also defined as the scaling factor $\mathrm{k}$ :

$$
k=\frac{R}{B_{a v e}} \frac{d B_{\text {ave }}}{d R}=\text { const }
$$

Integrating Eq. (44) yields:

$$
B_{\text {ave }}(R)=B_{0} \times\left(\frac{R}{R_{0}}\right)^{k}
$$

where $B_{0}$ is the vertical component of the magnetic field [23] averaged in azimuth for a given radius $R_{0}$. This formula describes the radial dependence of the azimuthally averaged field over the entire width of the magnet.

In conclusion, for a radial sector type FFAG, the vertical component of the magnetic field in the median plane can be written in the general form:

$$
B(R, \theta)=B_{0} \times\left(\frac{R}{R_{0}}\right)^{k} \times F(\theta)
$$

where $\mathrm{F}$ is the flutter function and $\mathrm{R}$ is the equivalent radius of the closed orbit as specified earlier. However,as will be shown below, this formula remains valid if we define $\mathrm{R}$ as the orbiting radius seen from the centre of the circular accelerator. The main assumption of this definition is that the function $\mathrm{B}(\mathrm{R}, \theta)$ is separable in radial and azimuthal coordinates, which will be discussed later. 
If $\mathrm{k}$ is fixed, then $R_{0}$ can be chosen arbitrarily: to show this, let's assume that $\mathrm{k}$ is fixed and does not have any radial dependence. Then for an arbitrary radius $R_{1}(\mathrm{Eq}$ 45) gives:

$$
B_{1}\left(R_{1}\right)=B_{0} \times\left(\frac{R_{1}}{R_{0}}\right)^{k}
$$

and injecting (Eq 47) into (Eq 45), one obtains:

$$
B_{\text {ave }}(R)=B_{1} \times\left(\frac{R}{R_{1}}\right)^{k}
$$

which proves the assertion above. From this, one can obtain the value of the average scaling factor of the KURRI 150 MeV FFAG, by computing the average z-component of the magnetic field in the median plane for different radii and by means of (Eq 49): in principle, if the magnets obey to the exact scaling law, $\mathrm{k}$ has to remain constant. However, due to imperfections, one can observe small variations in $\mathrm{k}$.

$$
k(R)=\frac{\log \left(\frac{B_{a v e}(R)}{B_{0}}\right)}{\log \left(\frac{R}{R_{0}}\right)}
$$

\section{KURRI $150 \mathrm{MeV}$ scaling FFAG and Benchmarking work}

Following the FFAG14 workshop held at BNL [13], a simulation campaign was established to benchmark several simulation codes. The main objective is to provide reliable modeling tools for FFAG type of accelerators and to better explain the results of the experiments at the KURRI $150 \mathrm{MeV}$ scaling FFAG [6]. Due to the non-linearities of such a machine, the beam dynamics are intricate to simulate and require careful modeling. The longitudinal and transverse beam dynamics in FFAGs differ significantly from the one in fixed orbit machines such as synchrotrons. A more detailed discussion about the correct modeling in FFAG can be found in [7].

\section{Design of the injection line into the KURRI FFAG:}

The first benchmarking test was carried out for the injection line into the main ring. The injection line transports the beam from a point outside the main ring to a matching point inside the ring suitable for the acceleration process. The

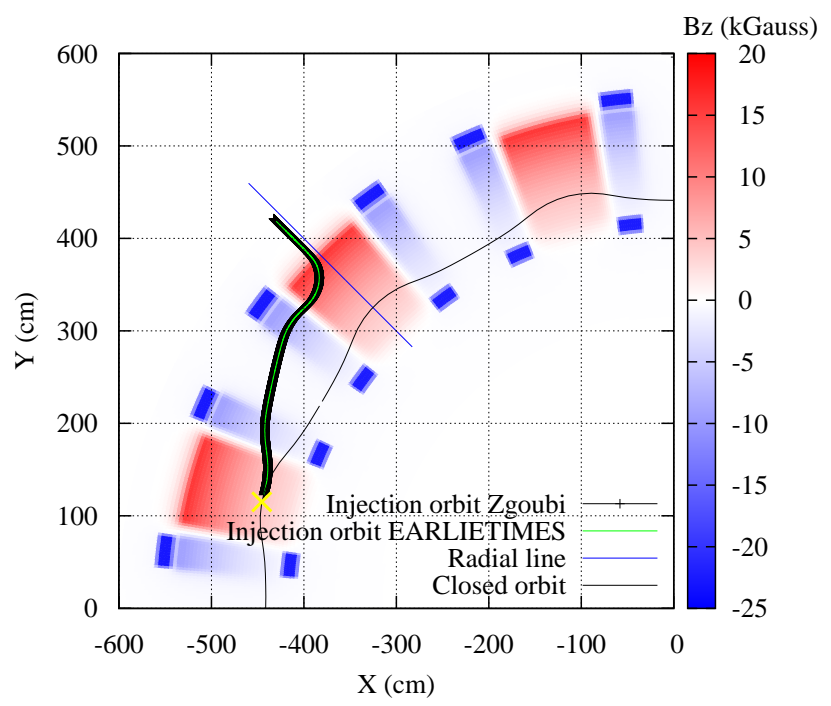

FIG. 1: Injection orbit into the main ring with the matching point (yellow). Only one quadrant is shown here. The two tracking codes, EARLIETIMES and ZGOUBI [19] show good agreement.

orbit suitable for acceleration is the first closed orbit shown in Fig. 1. The injected beam has to match the properties of this reference orbit at a given azimuth that in this case is the stripping foil, located where the injection orbit and the closed orbit intersect. An easy and straightforward solution to find the injection line is to track the beam backwards from the matching point. This approach was developed previously to design the injection line into the $800 \mathrm{MeV} /$ amu high power cyclotron [16]. 
The main property of this type of accelerator is that, in the ideal case, the number of betatron oscillations, also referred to as the tune, is energy-independent. This is guaranteed by the non-linearity of the field as explained earlier and avoids any resonance crossing during acceleration. Therefore, one important parameter for the benchmarking is the tune. Comparison of the tracking results with several codes show a good agreement (see Fig. 2). However, this demonstrated

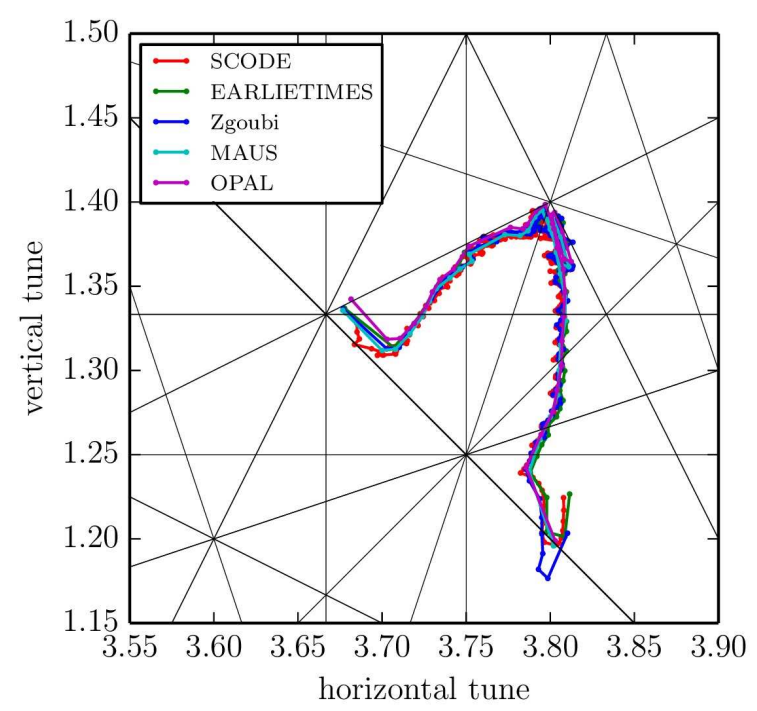

FIG. 2: Betatron tunes from 11 to $139 \mathrm{MeV}$ (left to right) calculated with several codes [6]. The Zgoubi model is in good agreement with the others.

that the tune variations are non-negligible (of the order of $15 \%$ in the vertical plane and $4 \%$ in the horizontal plane). The tune measurements are in agreement with this result.

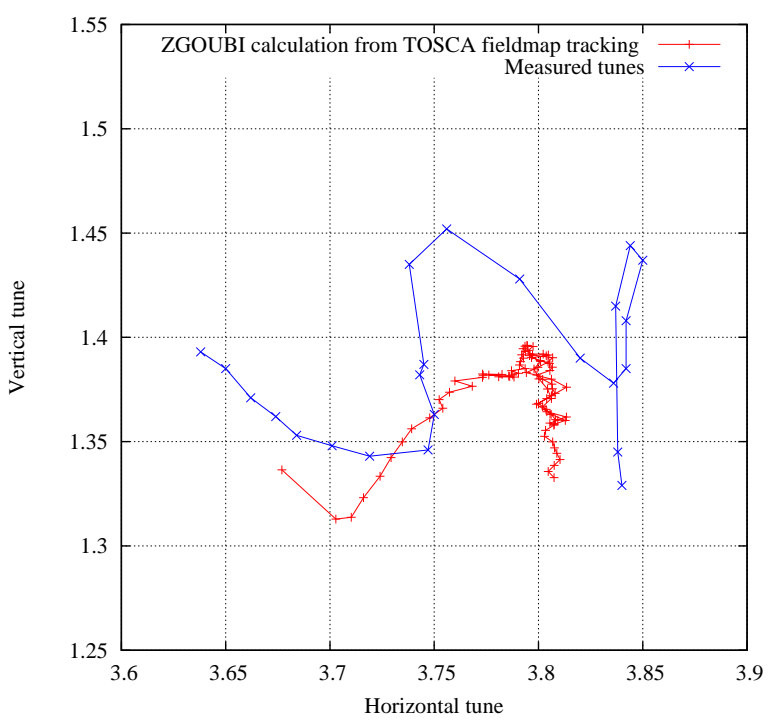

FIG. 3: Betatron tunes from 11 to $100 \mathrm{MeV}$ (left to right). The details of the measurement as well as the methods and tools that were used to characterize the $150 \mathrm{MeV}$ KURRI FFAG are discussed in [14]

This is investigated in detail in the last part of this paper.

Longitudinal beam dynamics comparison

Comparison of the longitudinal phase space showed good agreement between ZGOUBI and SOCDE (see Fig. 4). In the case of small synchrotron oscillations (the initial phase is close to the synchronous one), one can observe some differences 
essentially related to the initial conditions which are not the same in both codes. However, this is sufficient and useful when comparing multi-particle tracking results with slightly different settings.

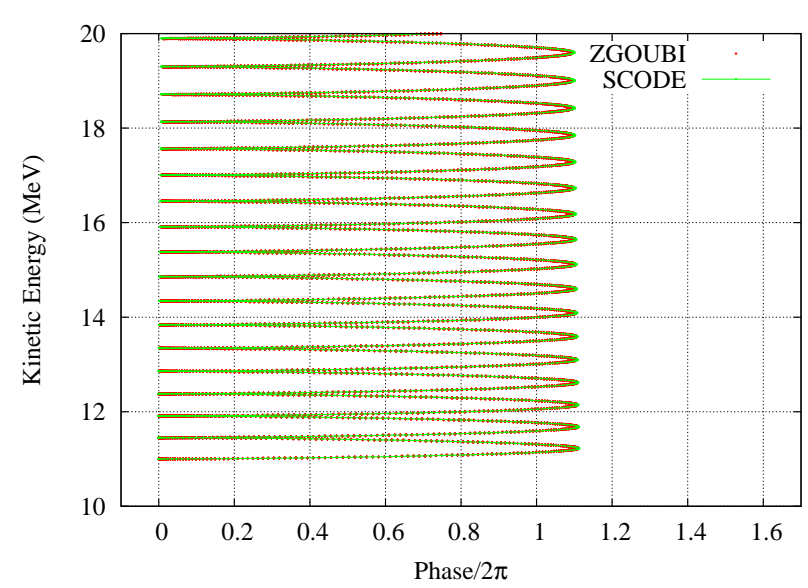

fig1The initial RF phase is 0 degree and the synchrotron oscillation is observed around the synchronous phase $\varphi_{s}=$ 30 deg.

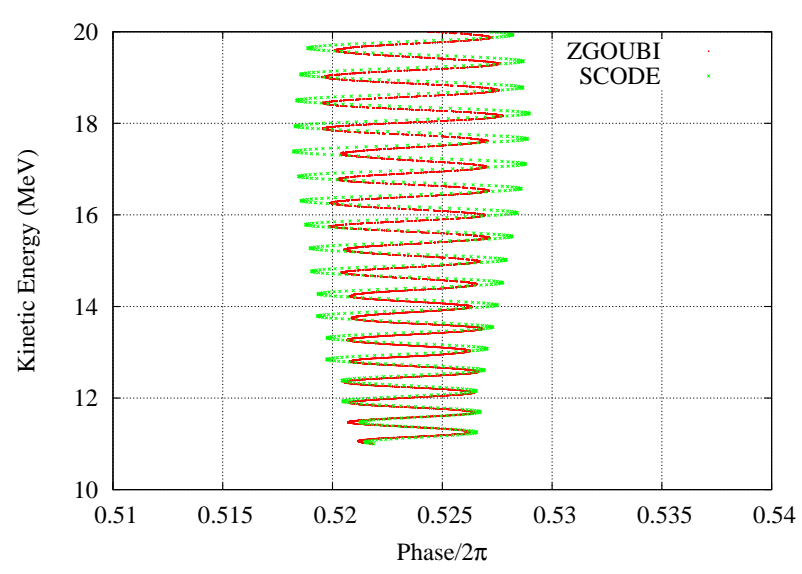

fig2The initial RF phase is 30 degree and the synchrotron oscillations are much smaller around the synchronous phase $\varphi_{s}=30 \mathrm{deg}$. The phase oscillations are susceptible to the initial conditions which are not exactly the same in the two codes.

FIG. 4: Comparison of the single particle trajectory in the longitudinal phase space.

Multi-particle tracking

This is an on-going work. Good agreement is observed between ZGOUBI and SCODE. However, this needs further investigation to explain the results.

The main objective of the following study is to explain the origin of the tune variations in the KURRI FFAG.

\section{Scaling factor analysis:}

Using the TOSCA field map ("TOSCA map f810 d1020.dat") of the KURRI machine one calculated the average magnetic field $\left(\frac{\int B d \theta}{\int d \theta}\right)$ for one sector. A plot of the z-component of the magnetic field for different radii is shown in (Fig鸟) below.

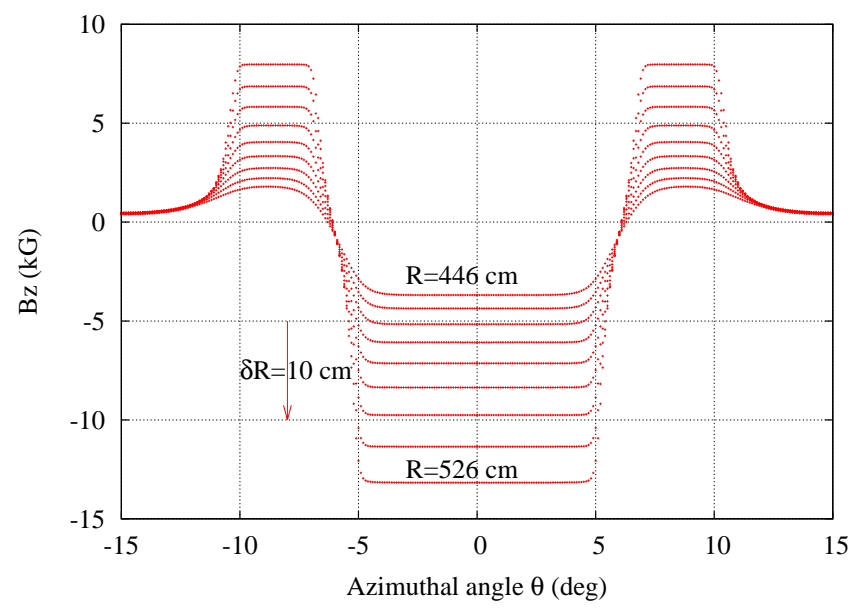

FIG. 5: Magnetic field for one sector of the ring obtained for different radii.

The average scaling factor is then deduced by applying Eq (49) where $R_{0}$ is the reference radius that in our case was chosen to be $R_{0}=445 \mathrm{~cm}$ (see Fig. (6). These results show about $18 \%$ error on the average scaling factor from injection to extraction (similar error for the tunes were observed). In order to understand the evolution of the scaling factor with the radius, one can apply the previous formula to evaluate the local scaling factor as a function of the radius for different azimuthal positions. The results are shown in (Fig. 7): for the different azimuthal angles below 4 degrees (namely: 0, 


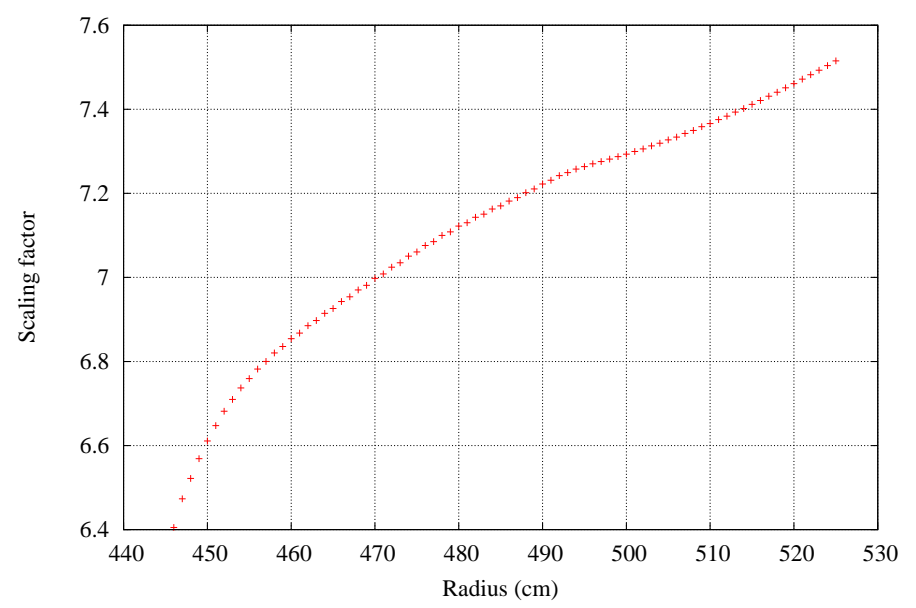

FIG. 6: Average scaling factor as a function of the radius (the reference radius is $R_{0}=445 \mathrm{~cm}$ ).

1.5 and 3 degrees), the local scaling factor (which is obtained by using the formula: $k\left(R, \theta=\theta_{0}\right)=\frac{\log \left(\frac{B\left(R, \theta=\theta_{0}\right)}{B_{0}}\right)}{\log \left(\frac{R}{R_{0}}\right)}$ ) is constant and varies from 7.4 to 7.6. This corresponds to the central region of the main focusing magnet where the magnetic field is constant (See Fig. 5). However, for the azimuthal positions above, the variations of the scaling factor seem to be random. In particular the fringe field region shows strong variations of the scaling factor. For the D-magnet, the variations of $\mathrm{k}$ are less obvious, although the average value is significantly higher than 7.6: all these considerations could explain the important tune variations that we will tackle more in detail when performing the beam stability analysis later on in this paper.

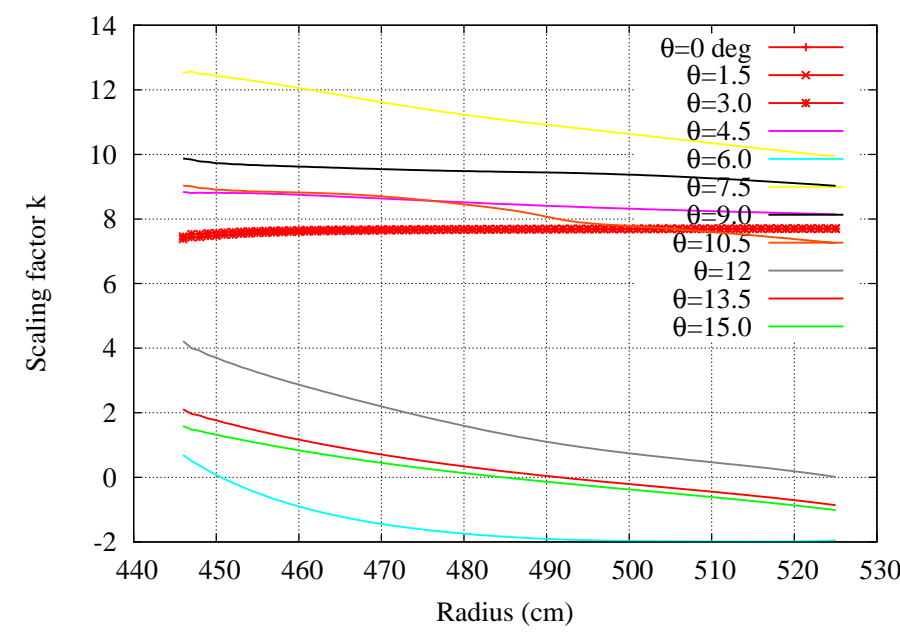

fig1Local scaling factor as a function of the radius for different azimuthal positions.

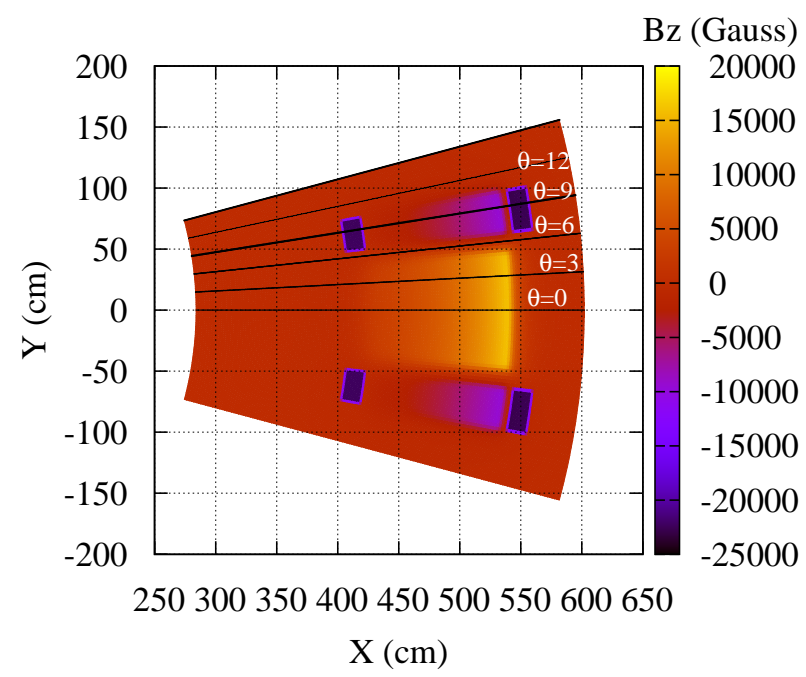

fig2Field map (for one sector in the median plane.

FIG. 7: Scaling factor as computed from the field map for different azimuthal positions.

So, now the assumption that the average scaling factor is constant must be withdrawn. This could explain the strong tune variations that were obtained before (and will be discussed later on). However, is the assumption that $R_{0}$ can be chosen arbitrarily in Eq. (45) still valid?

In order to answer this, let's rewrite Eq. (45) in the form

$$
B_{\text {ave }}(R)=B_{0} \times\left(\frac{R}{R_{0}}\right)^{k_{0}(R)}
$$

where now, we allow the radial variations of the scaling factor, $k_{0}(R)$.

If we choose our reference radius to be $R_{1}$ instead of $R_{0}$, then Eq. (50) rewrites:

$$
B_{\text {ave }}(R)=B_{1} \times\left(\frac{R}{R_{1}}\right)^{k_{1}(R)}
$$


If the assumption were to remain true, then $k_{0}(R)=k_{1}(R)$. However, we will derive an explicit transformation from $\left(R_{0}, B_{0}, k_{0}(R)\right)$ to $\left(R_{1}, B_{1}, k_{1}(R)\right)$ :

On the one hand, one has,

$$
B_{\text {ave }}(R)=B_{0} \times\left(\frac{R}{R_{0}}\right)^{k_{0}(R)}=B_{1} \times\left(\frac{R}{R_{1}}\right)^{k_{1}(R)}
$$

On the other hand,

$$
B_{1}=B_{0} \times\left(\frac{R_{1}}{R_{0}}\right)^{k_{0}\left(R_{1}\right)}
$$

And by injecting (Eq 53) into the first term of (Eq 52), one obtain:

$$
B_{\text {ave }}(R)=B_{1} \times\left(\frac{R_{0}}{R_{1}}\right)^{k_{0}(R 1)} \times\left(\frac{R}{R_{0}}\right)^{k_{0}(R)}
$$

Now, by applying the Logarithmic to these expressions, one obtain:

$$
\begin{aligned}
\log \left(B_{\text {ave }}(R)\right) & =\log \left(B_{1}\right)+k_{1}(R) \log \left(\frac{R}{R_{1}}\right) \\
& =\log \left(B_{1}\right)+k_{0}\left(R_{1}\right) \log \left(\frac{R_{0}}{R_{1}}\right)+k_{0}(R) \log \left(\frac{R}{R_{0}}\right)
\end{aligned}
$$

so that,

$$
k_{1}(R)=\frac{\log \left(\frac{R}{R_{0}}\right)}{\log \left(\frac{R}{R_{1}}\right)} \times k_{0}(R)+\frac{\log \left(\frac{R_{0}}{R_{1}}\right)}{\log \left(\frac{R}{R_{1}}\right)} \times k_{0}\left(R_{1}\right)
$$

It results from this equation that:

- If $k_{0}(R)=k_{0}\left(R_{1}\right)=$ const $\Longrightarrow k_{1}(R)=k_{0}(R)$, which was proved in the first paragraph.

- If $R_{0}=R_{1} \Longrightarrow k_{0}\left(R_{0}\right)=k_{1}\left(R_{1}\right)$ which is trivial.

- If $R=R_{0} \Longrightarrow k_{1}\left(R_{0}\right)=k_{0}\left(R_{1}\right)$ which can be easily verified from (Fig 8 ).

And more generally: $\forall(i, j) \in \mathbb{N} \times \mathbb{N}, k_{i}\left(R_{j}\right)=k_{j}\left(R_{i}\right)$ where $R_{i}$ and $R_{j}$ are reference radii for $k_{i}$ and $k_{j}$ respectively.

In order to check this result, we compute the average scaling factor by varying the reference radius $R_{0}$ in the previous Equation (50). The results are shown in Fig. 8 .

In conclusion, $\mathrm{k}(\mathrm{R})$, as defined earlier, is a relative quantity and cannot be defined in an absolute way (unless it is constant). So a reference radius in the field map has to be well defined with respect to which the scaling factor can be determined. Yet how to calculate $\mathrm{k}(\mathrm{R})$ in a reliable way? Also, is there a reference radius that can be chosen in order to obtain correct results?

\section{A more accurate way to compute the average scaling factor:}

Let's use the original (correct) definition of the scaling factor:

$$
k=\frac{d B / B}{d R / R}
$$

where $\mathrm{B}$ is the field averaged in azimuth (sometines $\mathrm{k}$ is also referred to as the mean field index). This form has the merit that it does not have any assumption as to the form of the scaling factor, whether it is constant or is a function of R. Thus it is more rigorous to apply in order to compute k. From the field map, one applied Eq. (58) below:

$$
k\left(R_{i}\right)=\frac{\frac{B_{i+1}-B_{i}}{B_{i}}}{\frac{R_{i+1}-R_{i}}{R_{i}}}
$$

where $R_{i+1}-R_{i}$ is limited by the mesh size (here $R_{i+1}-R_{i}=1 \mathrm{~cm}$ ). The result is shown in Fig. (9). The correct scaling 


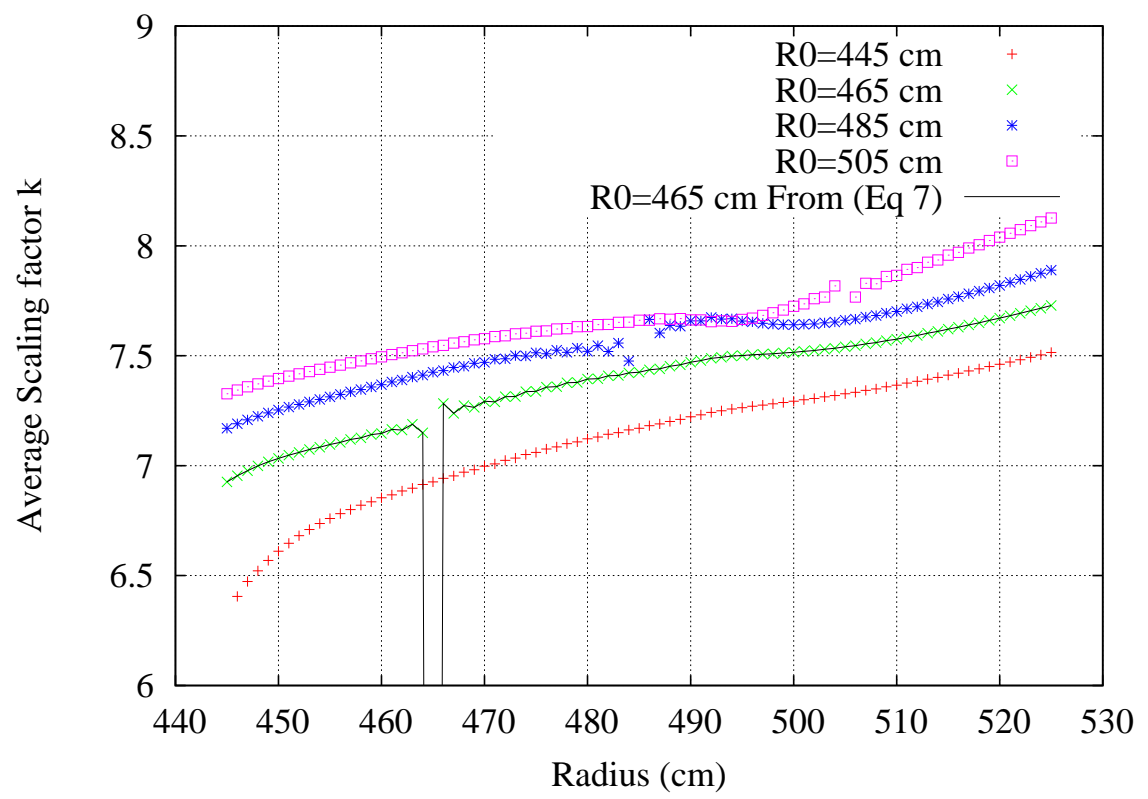

FIG. 8: Average scaling factor for different reference radii $R_{0}$ : Eq. (56) above was applied to transform $\left(R_{0}=445 \mathrm{~cm}, B_{0}, k_{0}(R)\right)$ into $\left(R_{1}=465 \mathrm{~cm}, B_{1}, k_{1}(R)\right)$ which gave perfect agreement with the result obtained from the field map. The discontinuity point simply corresponds to the case where $\mathrm{R}=R_{1}$ for which the formula is not defined. (all the other possible transformations in this plot were also checked and gave perfect agreement as expected).

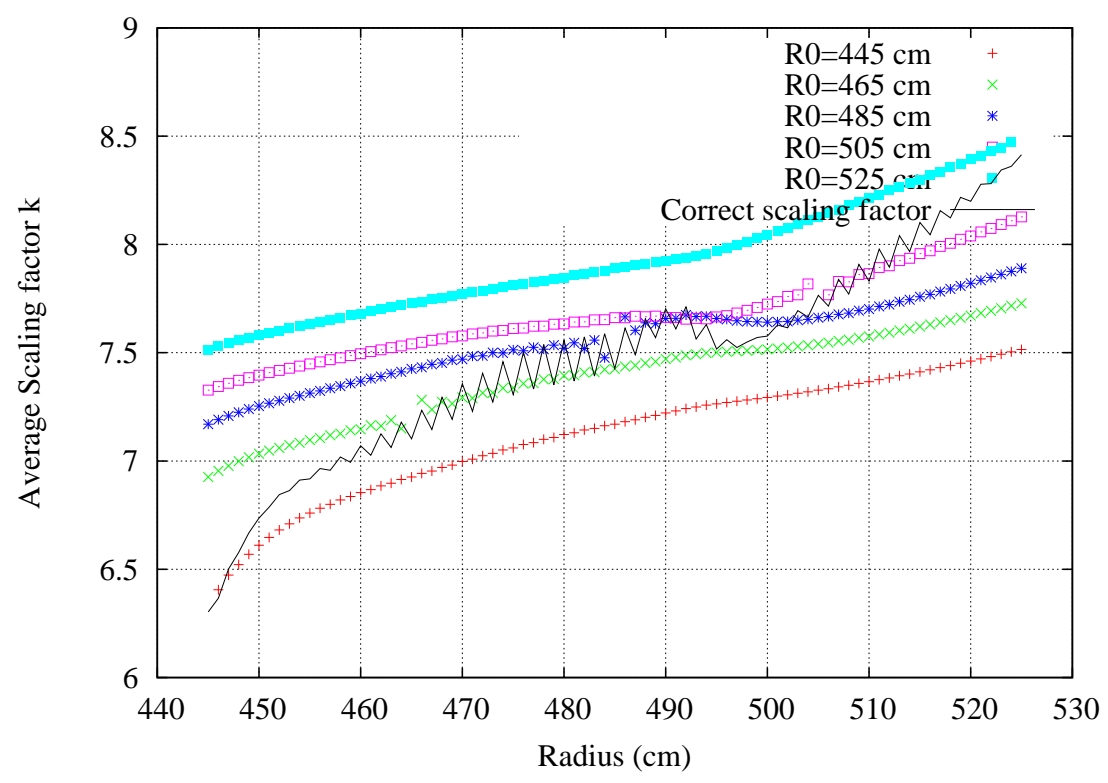

FIG. 9: Average scaling factor for different reference radii $R_{0}$.

factor (curve in black) was obtained from Eq. (58) where no assumption is made as to its form: it can be seen that each one of the other curves which were obtained from the definition specified in Eq. (49) is valid only in the vicinity of the reference radius $R_{0}$ where the variations of $\mathrm{k}$ are still not too important.

Conclusion: The radial scaling law $B(R)=B_{0} \times\left(\frac{R}{R_{0}}\right)^{k(R)}$ is only valid in the vicinity of the reference radius $R_{0}$, simply because it is not a correct general solution of Eq. (577). Thus, when defining this equation, one has to choose $R_{0}$ carefully. However, as will be shown later, this form is particularly useful to simplify the analysis when writing the equations of motion in the vicinity of each of the closed orbits.

\section{The average scaling factors for the $\mathrm{F}$ and $\mathrm{D}$ magnets:}

The correct definition of $\mathrm{k}$ (see Eq.(57)) was applied again in order to compute the exact values of $\mathrm{k}$ for the focusing and defocusing magnets individually (the average value of the field B is taken over the width of each of these magnets). The results are shown in Fig. (10). For the F-magnet, the variations of $\mathrm{k}$ are not important, however for the D-magnet, one observes that the scaling factor changes significantly, and its average value is $\mathrm{k} \approx 9.1$. 


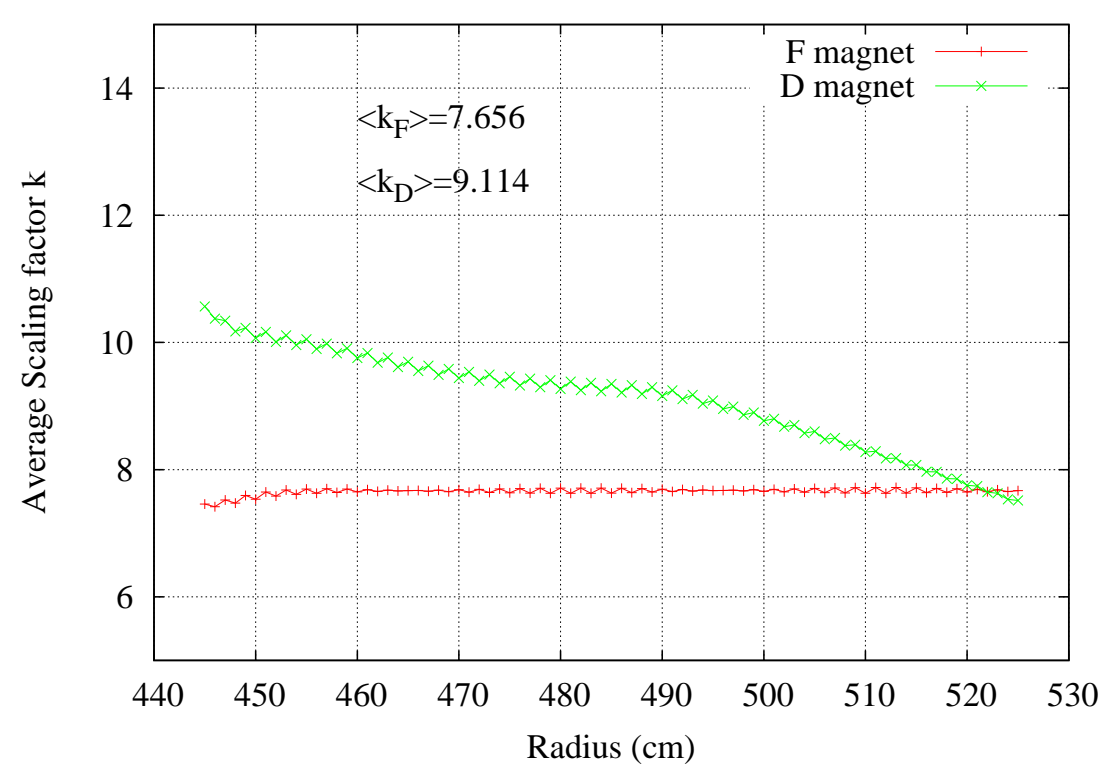

FIG. 10: Average scaling factor for the $\mathrm{F}$ and $\mathrm{D}$ magnets.

Conclusion: The scaling factor of the F-magnet is constant and $\left\langle k_{F}>\approx 7.656\right.$. Yet for the D-magnet, the variations are non negligible and $\left\langle k_{D}>\approx 9.114\right.$.

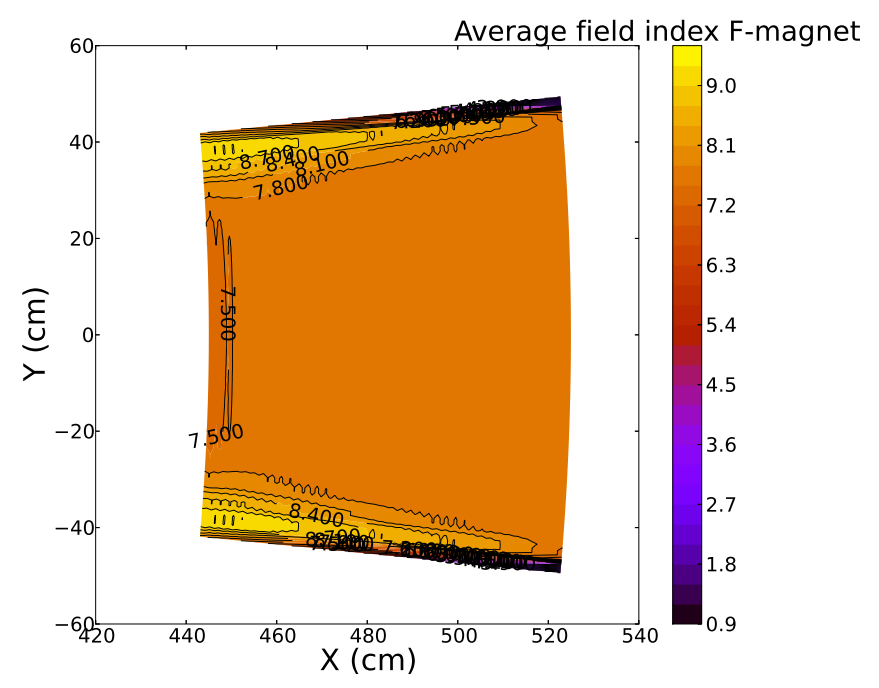

fig1Average field index map of the focusing magnet $\left(k_{F}\right)$

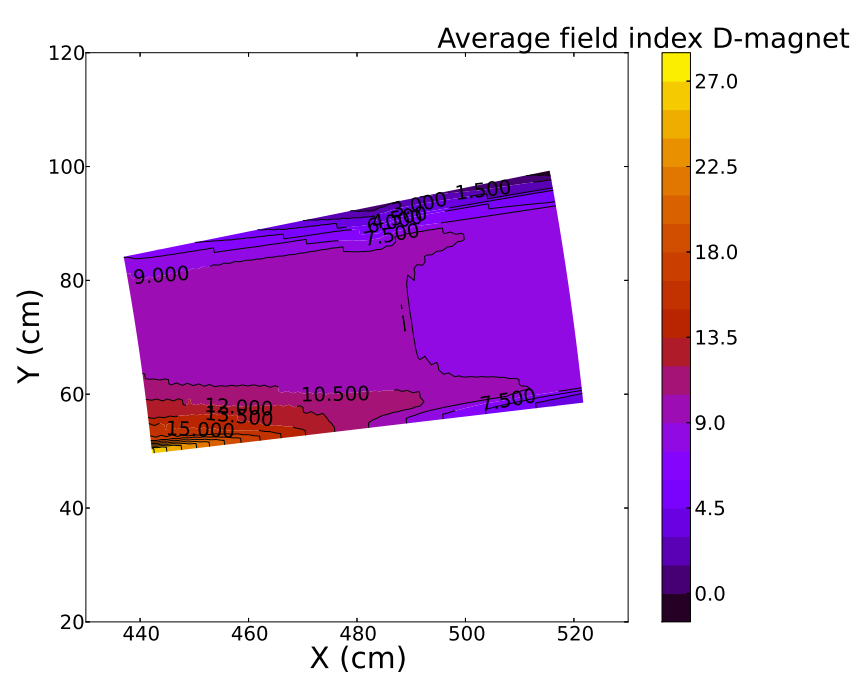

fig2Average field index map of the defocusing magnet

$\left(k_{D}\right)$

FIG. 11: Contour plot of the average field indices $k_{F}$ and $k_{D}$ : as can be observed from this plot, there is a cross-talk between the two magnets mainly in the interaction region of the DFD triplet.

In order to understand the origin of these strong variations, mainly in the defocusing magnet, we compute the map of the average field index of the two magnets. As can be seen in Fig. 11, the main source of discrepancy is observed in the interaction region between the magnets and seems to mostly affect the defocusing one. This effect is mostly dominant in the injection region.

Next, we develop an analytical model that takes into account the scaling imperfections of the magnetic field.

\section{Analytical solution of the magnetic field to account for scaling imperfections:}

The average field index $\mathrm{k}$, is defined by:

$$
k=\frac{d B / B}{d R / R}
$$

where $\mathrm{B}$ is the field averaged in azimuth for a radius $\mathrm{R}$. Thus $\mathrm{k}$ is a function of the radius. Based on the previous calculation of the scaling factor, it turns out that $\mathrm{k}$ is a function of $\mathrm{R}$ so that the previous form of the magnetic field (see 
Eq. (45)) is not quite accurate to describe the magnets. In order to obtain the new form of the magnetic field, while keeping the same definition of the scaling factor, let's assume that $\mathrm{k}$ can be fitted with an n-order polynomial which is a function of the radius: $\mathrm{k}$ has the form

$$
k(R)=\sum_{i=0}^{n} a_{i}\left(\frac{R}{R_{0}}\right)^{i}
$$

Then by equating Eq. (59) and Eq. (60), one obtains:

$$
\frac{d B}{B}=\sum_{i=0}^{n} \frac{a_{i}}{R_{0}^{i}} R^{i-1} d R=a_{0} \frac{d R}{R}+\sum_{i=1}^{n} \frac{a_{i}}{R_{0}^{i}} R^{i-1} d R
$$

which gives after integration:

$$
\begin{gathered}
\ln \left(\frac{B}{B_{0}}\right)=a_{0} \ln \left(\frac{R}{R_{0}}\right)+\sum_{i=1}^{n} \frac{a_{i}}{R_{0}^{i}} \int_{R_{0}}^{R} R^{i-1} d R \\
B(R)=B_{0} \exp \left[a_{0} \ln \left(\frac{R}{R_{0}}\right)+\sum_{i=1}^{n} \frac{a_{i}}{R_{0}^{i}} \frac{\left(R^{i}-R_{0}^{i}\right)}{i}\right]
\end{gathered}
$$

so that the general form of the magnetic field becomes, $B(R, \theta)=B(R) F(\theta)$, i.e,

$$
B(R, \theta)=B_{0}\left(\frac{R}{R_{0}}\right)^{a_{0}} \times \exp \left(\sum_{i=1}^{n} a_{i} \frac{R^{i}-R_{0}^{i}}{i \times R_{0}^{i}}\right) \times F(\theta)
$$

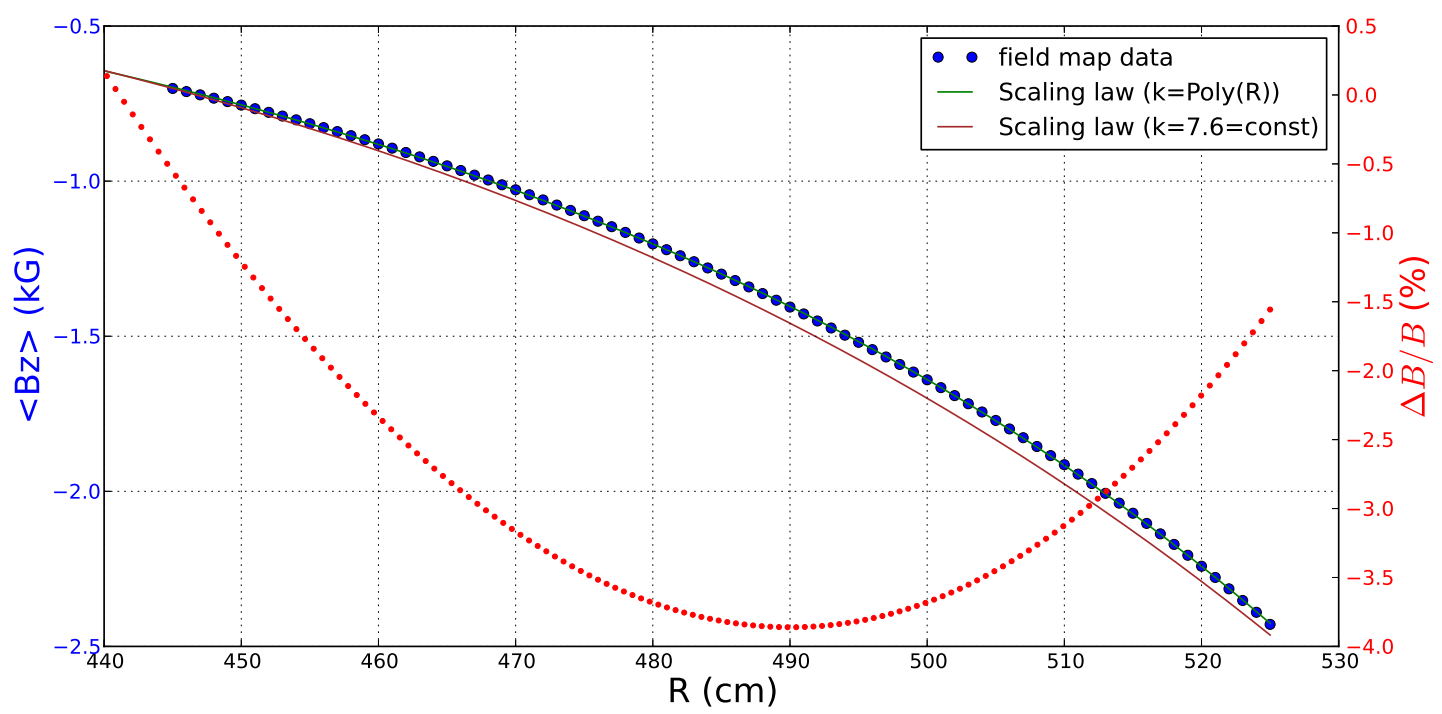

FIG. 12: Average magnetic field as a function of the radius: the field map results are shown in blue while the scaling laws with $\mathrm{k}$ constant and variable are shown in red and green respectively. The constant k model shows some discrepancy with the field map data $(|\Delta B / B| \leq 4 \%)$.

The new scaling law takes into account the R-dependence of the scaling factor and thus is more accurate to describe the magnet as can be seen from Fig. 12. The final parameters of the fitting are: $\mathrm{n}=3, a_{0}=7.6, a_{1}=4.74 e-3$, $a_{2}=-5.39 e-5, a_{3}=9.04 e-8$ and $R_{0}=480 \mathrm{~cm}$.

\section{Flutter function:}

If $\mathrm{B}(\mathrm{R}, \theta)$ were separable in radial and azimuthal coordinates, then one should obtain:

$$
F(\theta)=\frac{B(R, \theta)}{<B>(R)}
$$

Yet, when computing $\mathrm{F}(\theta)$ for various radii (see Fig. 13), one observed that $\mathrm{F}$ has also a radial dependence. This proves that $\mathrm{B}(\mathrm{R}, \theta)$ is not a separable function. 


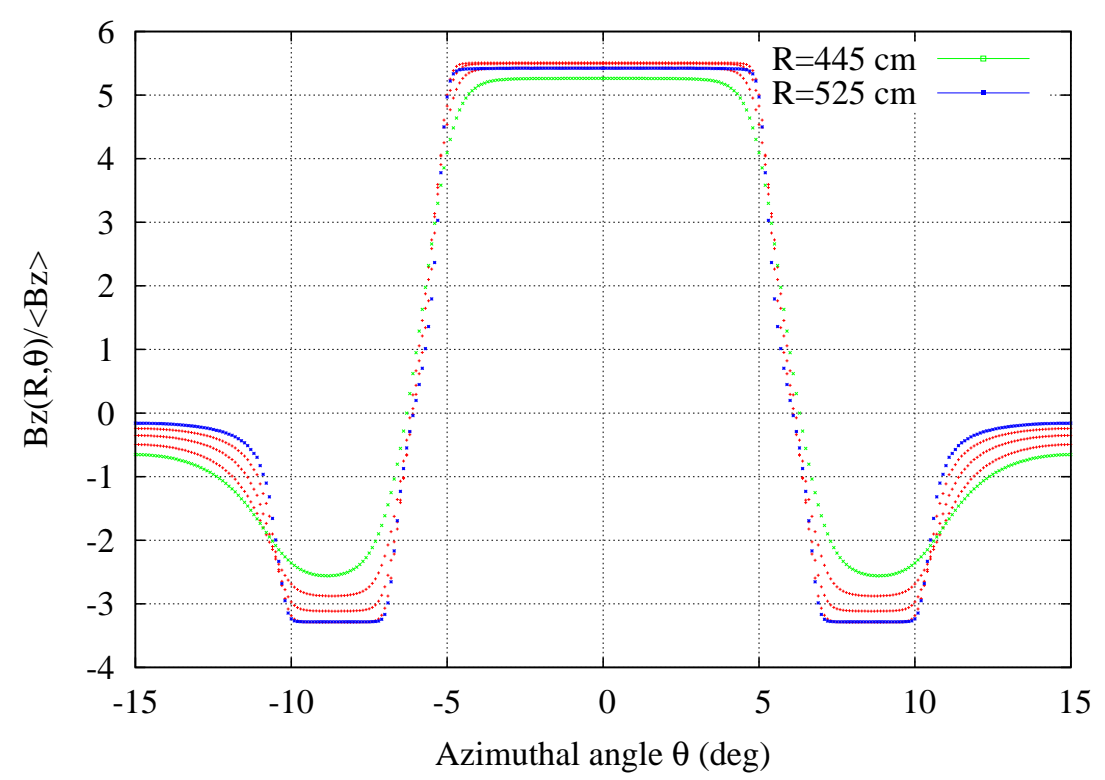

FIG. 13: Flutter function $\mathrm{F}$ as a function of the azimuthal angle $\theta$ for different radii.

In conclusion, the general form of the magnetic field is:

$$
\begin{array}{r}
B(R, \theta)=<B>(R) \times F(R, \theta) \\
F(R)=<F(R, \theta)>=1
\end{array}
$$

In order to better understand the radial dependence of the flutter, we start from Eq. (62) where we evaluate the field along a closed orbit (instead of a fixed radius orbit). We introduce the transformation: $R(\theta)=<R>\times(1+r(\theta)$ ) where $r(\theta)$ is a periodic function describing the periodic oscillation around the fixed radius orbit $\langle R\rangle$. This yields:

$$
B(R(\theta), \theta)=B_{0}\left(\frac{<R>}{R_{0}}\right)^{a_{0}} \times[1+r(\theta)]^{a_{0}} \times \exp \left(\sum_{i=1}^{n} a_{i} \frac{R(\theta)^{i}-R_{0}^{i}}{i \times R_{0}^{i}}\right) \times F(\theta)
$$

The above equation is only valid under the assumption that all orbits are parallel to each other. Otherwise $r(\theta)$ needs to be re-calculated for each orbit.

-If the scaling factor is constant (i.e. R-independent), then $a_{i}=0 \quad \forall i \geq 1$ and Eq. (66) can be written in the form:

$$
\begin{aligned}
B(R(\theta), \theta) & =B(<R>) \times F_{1}(\theta) \\
F_{1}(\theta) & =[1+r(\theta)]^{a_{0}} \times F(\theta)
\end{aligned}
$$

This shows that the flutter function of the equilibrium orbit is constant and differs from that of the fixed radius orbit through a simple formula. This is only true if there are no scaling imperfections.

\section{TUNE CALCULATION AND BEAM STABILITY ANALYSIS}

Technically, it is impossible to make a field which corresponds exactly to the designed one. Therefore, it is important to understand the effect of small imperfections of the field on the beam dynamics.

The general equations of motion including non-linear terms and imperfections are defined by:

$$
\left\{\begin{array}{l}
\frac{d^{2} x}{d s^{2}}=P(x, y, s) \\
\frac{d^{2} y}{d s^{2}}=Q(x, y, s)
\end{array}\right.
$$

where $\mathrm{x}$ and $\mathrm{y}$ represent the deviation around the closed orbit, and $\mathrm{P}$ and $\mathrm{Q}$ are real analytic functions of $\mathrm{x}, \mathrm{y}$ and $\mathrm{s}$. In the following, we investigate the stability of the particle trajectories that can arise with different possible pairs of scaling factors $\left(k_{F}, k_{D}\right)$. We use three different approaches to investigate the beam stability due to field errors: the first approach is based on the hard edge model: we assume that the magnetic field drops abruptly to zero at the edges of the magnet which greatly simplifies the analysis and allows the development of a geometrical model of the 
lattice. The second model is an analytical model which we use to seek an approximate solution of the particle equations of motion. The last and third model is the ZGOUBI tracking model of the magnet, which is the most accurate one. The ZGOUBI model solves the non-linear equation of motion using field maps or user-implemented analytical models. To conclude, we establish a comparison between the different results and comment on the outcome of this study.

\section{Hard edge model}

In this first approach, we restrict our analysis to a linear motion around the closed orbits. Therefore, Eqs. (68) simplify to:

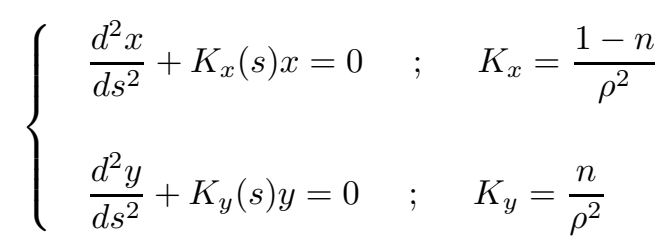

which is the set of linearized equations with periodic coefficients valid in a small neighborhood of the equilibrium orbit. There is a different linearized set of equations for each closed orbit, i.e different pairs $\left(K_{x}, K_{y}\right)$ so that a solution of the above equations may not be generalized to the whole system. For the following stability analysis, we prefer to use the term "stability in the vicinity of the closed orbit", to recall that a linear system is only a first approximation to the general problem. However, for small perturbations, the stability of a non-linear system may often be inferred from the stability of its associated linear system. To make the calculations tractable, we assume that the field inside each magnet is constant with respect to the azimuth and drops abruptly to zero at the edges as illustrated in Fig. 14. Also, we keep the radial dependence of the field ( $\mathrm{k}$ is $\mathrm{R}$-dependent) and allow the scaling law to be different between the $\mathrm{F}$ and D-magnet.

We start from the field index $\mathrm{n}$ where we neglect the scalloping of the orbits,

$$
n=-\frac{\rho}{B} \frac{d B}{d x} \approx-\frac{\rho}{B} \frac{d B}{d R}=-\frac{\rho}{R} k
$$

Now, we use the index $\mathrm{i}$ to make the distinction between the F and D magnet and authorize the field index to change with the radius. This yields,

$$
n_{i}\left(R_{i}\right)=-\frac{\rho_{i}}{R_{i}} k_{i}\left(R_{i}\right) \quad ; \quad i=F / D
$$

In order to solve the equation of motion, we use a piecewise method that consists in following the particle motion through a series of elements defined by their transfer matrices (Focusing/Defocusing magnets and drifts). This requires the development of the geometrical model to obtain the relationship between $\rho_{i}$ and $R_{i}$, therefore the field index $n_{i}$.

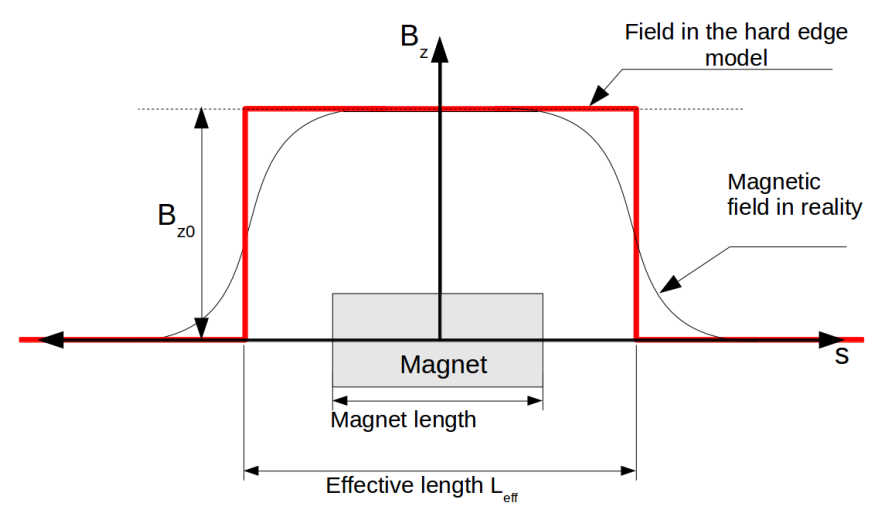

FIG. 14: Magnetic field in the hard edge model.

For the focusing magnet, $K=K_{x, y}>0$ :

$$
\mathbf{M}_{\mathrm{foc}}=\left(\begin{array}{cc}
\cos (\sqrt{K} s) & \frac{1}{\sqrt{K}} \sin (\sqrt{K} s) \\
-\sqrt{K} \sin (\sqrt{K} s) & \cos (\sqrt{K} s)
\end{array}\right)
$$

For the defocusing magnet, $K=K_{x, y}<0$ :

$$
\mathbf{M}_{\mathrm{defoc}}=\left(\begin{array}{cc}
\cosh (\sqrt{|K|} s) & \frac{1}{\sqrt{|K|}} \sinh (\sqrt{|K|} s) \\
\sqrt{|K|} \sinh (\sqrt{|K|} s) & \cosh (\sqrt{|K|} s)
\end{array}\right)
$$


The transfer matrices, both horizontal and vertical for half the sector can then be computed:

$$
\begin{aligned}
& M_{\text {sector } / 2}^{x}=M_{F / 2}^{x} \times M_{\text {edge }, F}^{x} \times M_{\text {drift } 1} \times M_{\text {edge } 1, D}^{x} \times M_{D}^{x} \times M_{\text {edge }, D}^{x} \times M_{\text {drift } / 2} \\
& M_{\text {sector } / 2}^{y}=M_{F / 2}^{y} \times M_{\text {edge }, F}^{y} \times M_{\text {drift } 1} \times M_{\text {edge } 1, D}^{y} \times M_{D}^{y} \times M_{\text {edge }, D}^{y} \times M_{\text {drift } / 2}
\end{aligned}
$$

The number of betatron oscillations per cell $\nu_{x, y}$ can then be calculated using:

$$
\cos \left(2 \pi . \nu_{x, y}\right)=\frac{1}{2} \operatorname{Tr}\left(M_{\text {sector }}^{x, y}\right)
$$

and a condition sine qua non for the stability of the transverse motion is:

$$
\left|\operatorname{Tr}\left(M_{\text {sector }}^{x, y}\right)\right|<2
$$

Now, we set the geometrical model of the DFD triplet (see Fig. 15) and express all relations as a function of the bending radius $\rho_{F}$ of the focusing magnet:

\section{Bz (kGauss)}

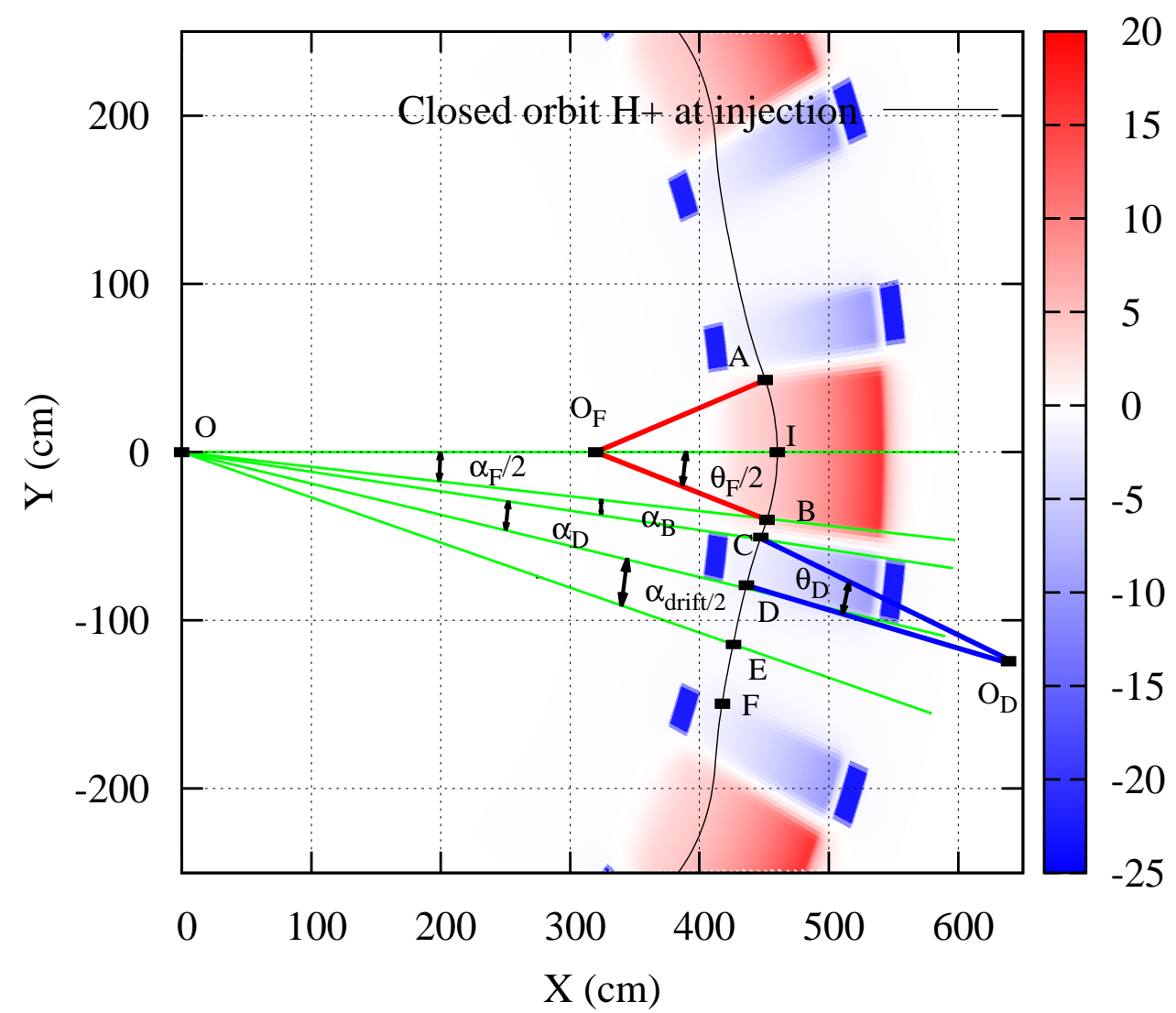

FIG. 15: Example of trajectory in the hard edge model of a KURRI-like DFD triplet.

$$
\begin{aligned}
\rho_{F}=O_{F} A=O_{F} I=O_{F} B & ; \quad \rho_{D}=O_{D} C=O_{D} D \quad ; \quad O C \neq O D \\
R_{F}=O I & ; R_{D}=O C \quad ; \quad L_{B}=B C \quad ; \quad L_{\text {drift }}=D F=2 D E \\
(B C) \perp\left(O_{F} B\right) & ; \quad(B C) \perp\left(O_{D} C\right) \quad ; \quad\left(O_{D} D\right) \perp(D E) \\
\frac{\alpha_{F}}{2}+\alpha_{B}+\alpha_{D}+\frac{\alpha_{\text {drift }}}{2}=\frac{\pi}{N} & ; \quad \theta_{F}-2 \theta_{D}=\frac{2 \pi}{N} \quad ; \quad \theta_{F}, \theta_{D}>0 .[24]
\end{aligned}
$$

Applying the sinus law in the triangle $O O_{F} B$ yields:

$$
\frac{\rho_{F}}{R_{F}}=\frac{\sin \left(\frac{\alpha_{F}}{2}\right)}{\sin \left(\frac{\alpha_{F}}{2}\right)+\sin \left(\frac{\theta_{F}-\alpha_{F}}{2}\right)}
$$

[24] $(\widehat{\overrightarrow{O I}, \overrightarrow{O E}})=\pi / N=\left(\widehat{O_{F}, \widehat{O_{F} B}}\right)+\left(\widehat{O_{D} \vec{C}, \bar{O}_{D} D}\right)=\theta_{F} / 2-\theta_{D} \quad ; \quad \theta_{F}, \theta_{D}>0$ 
Applying the sinus law in the triangles $O B I, O_{F} B I$ and $O B C$ yields:

$$
\frac{L_{B}}{\rho_{F}}=\frac{L_{B}}{O B} \times \frac{O B}{\rho_{F}}=\frac{\sin \left(\alpha_{B}\right)}{\cos \left(\frac{\theta_{F}-\alpha_{F}}{2}-\alpha_{B}\right)} \times \frac{\sin \left(\frac{\theta_{F}}{2}\right)}{\sin \left(\frac{\alpha_{F}}{2}\right)}
$$

Applying the sinus law in the triangle $O B C$ yields:

$$
\frac{R_{D}}{L_{B}}=\frac{\cos \left(\frac{\theta_{F}-\alpha_{F}}{2}\right)}{\sin \left(\alpha_{B}\right)}
$$

Applying the sinus law in the triangles $O C D$ and $O_{D} C D$ yields:

$$
\frac{\rho_{D}}{R_{D}}=-\frac{\sin \left(\alpha_{D}\right)}{\cos \left(\alpha_{D}+\alpha_{B}+\frac{\theta_{D}-\theta_{F}+\alpha_{F}}{2}\right)} \times \frac{\cos \left(\frac{\theta_{D}}{2}\right)}{\sin \left(\theta_{D}\right)}
$$

Applying the sinus law in the triangles $O D E$ and $O C D$ yields:

$$
\frac{L_{d r i f t}}{R_{D}}=\frac{L_{d r i f t}}{O D} \times \frac{O D}{R_{D}}=2 \sin \left(\frac{\alpha_{d r i f t}}{2}\right) \times \frac{\cos \left(\alpha_{B}+\frac{\theta_{D}-\theta_{F}+\alpha_{F}}{2}\right)}{\cos \left(\alpha_{D}+\alpha_{B}+\frac{\theta_{D}-\theta_{F}+\alpha_{F}}{2}\right)}
$$

\section{Some remarks:}

- The second cardinal condition of the similarity (see Eq (43)) implies that the ratio $\rho_{i} / R_{i}$ is constant and shall be the same for different closed orbits. This necessarily implies that the bending angles $\theta_{F}$ and $\theta_{D}$ are constant and all the relations established above as well.

-Whenever the scaling factors $k_{F}$ and $k_{D}$ are different, the similarity of the orbits is no longer satisfied. To show this, let's proceed using a reductio ad absurdum: let's suppose that $\rho_{i} / R_{i}=$ const. Then, from Eq (78) to (81), it results that $\rho_{F} / \rho_{D}=$ const and $R_{F} / R_{D}=$ const. However, if we write,

$$
\left\{\begin{array}{l}
B \rho=\frac{p}{q}=B_{F} \rho_{F}=B_{D} \rho_{D} \\
B_{F}\left(R_{F}\right)=B_{F 0}\left(\frac{R_{F}}{R_{0}}\right)^{k_{F}} \\
B_{D}\left(R_{D}\right)=B_{D 0}\left(\frac{R_{D}}{R_{0}}\right)^{k_{D}}
\end{array}\right.
$$

this yields,

$$
\begin{aligned}
\frac{\rho_{F}}{\rho_{D}} & =\frac{B_{D}}{B_{F}}=\frac{B_{D 0}}{B_{F 0}} \times \frac{R_{D}^{k_{D}}}{R_{F}^{k_{F}}} \times R_{0}^{k_{F}-k_{D}} \quad ; \quad R_{D} \propto R_{F} \\
& \propto R_{F}^{k_{D}-k_{F}}
\end{aligned}
$$

which is R-dependent if $k_{F} \neq k_{D}$. This is in contradiction with $\rho_{F} / \rho_{D}=$ const, which proves the assertion above.

Now, we distinguish two cases:

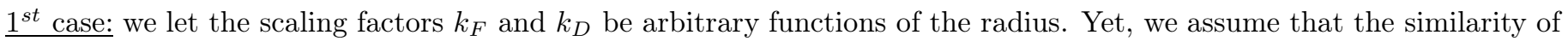
the orbits remains, i.e $\rho_{i} / R_{i} \approx$ const. From Eq (71) it results that $n_{F}\left(R_{F}\right) \propto k_{F}\left(R_{F}\right)$ and $n_{D}\left(R_{D}\right) \propto k_{D}\left(R_{D}\right)$. This is generally a good approximation if $k_{F}$ and $k_{D}$ are slightly different.

Based on that, the transfer matrices of the cell can be obtained and the tunes computed using Eq (75).

In the original paper of Symon in 1956, it was shown that the number of betatron oscillations per turn obtained from the smooth approximation are given by [8]:

$$
\begin{aligned}
& \nu_{x}^{2}=k+1+\frac{(k+1)^{2} f^{2}}{N^{2}}<g_{1}^{2}>_{A v} \\
& \nu_{y}^{2}=-k+\frac{f^{2}}{2}+\frac{(k-1)^{2} f^{2}}{N^{2}}<g_{1}^{2}>_{A v}
\end{aligned}
$$


where $\mathrm{k}$ is the average scaling factor that is supposed to remain constant. However, this does not take into account any scaling imperfection. Therefore, one decided to extend the previous result by making the following conjecture:

$$
\begin{aligned}
\nu_{x}^{2} & =x_{1} k_{F}+x_{2} k_{D}+\left(x_{3} k_{F}+x_{4} k_{D}+x_{5}\right)^{2} \\
\nu_{y}^{2} & =y_{1} k_{F}+y_{2} k_{D}+y_{3}
\end{aligned}
$$

where $\left(x_{1}, x_{2}, x_{3}, x_{4}, x_{5}\right)$ and $\left(y_{1}, y_{2}, y_{3}\right)$ are constants that are strictly related to the geometry of the cell.

If the conjecture were true, then solving a system of 5 non-linear equations in the horizontal plane, and a system of 3 linear equations in the vertical plane, will provide for a given cell the horizontal and vertical tunes, respectively. For instance, we solve the following system in the vertical plane,

$$
\left\{\begin{array}{l}
k_{F 1} y_{1}+k_{D 1} y_{2}+y_{3}=\nu_{y 1}^{2} \\
k_{F 2} y_{1}+k_{D 2} y_{2}+y_{3}=\nu_{y 2}^{2} \\
k_{F 3} y_{1}+k_{D 3} y_{2}+y_{3}=\nu_{y 3}^{2}
\end{array}\right.
$$

where $\left(k_{F i}, k_{D i}\right)_{i=1,3}$ are provided to the hard edge model implemented in Mathematica and $\left(\nu_{y i}\right)_{i=1,3}$ are calculated using Eq (75). This yields $\left(y_{1}, y_{2}, y_{3}\right)=(-1.1777,0.5859,9.1117)$. For the horizontal plane, we proceed in the same way and obtain $\left(x_{1}, x_{2}, x_{3}, x_{4}, x_{5}\right)=(2.4990,-0.4833,-0.5262,-0.0121,3.1184)$. The results are finally summarized in Fig. 16.

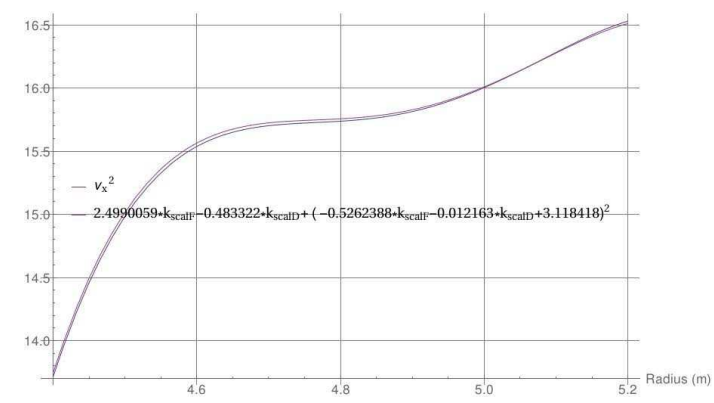

fig1Horizontal tune of the cell as a function of the radius.

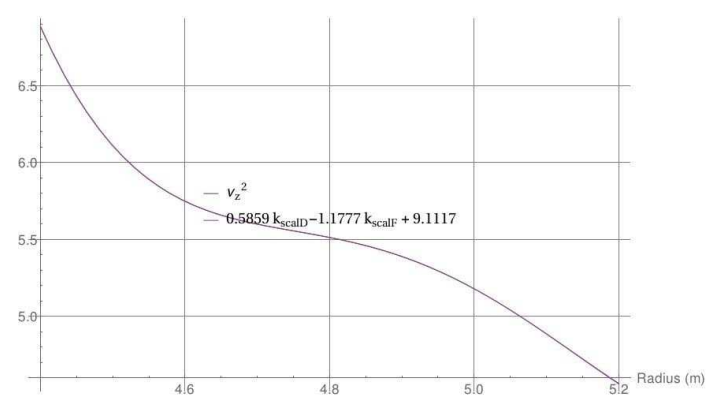

fig2Vertical tune of the cell as a function of the radius.

FIG. 16: Comparison of the tune calculation using the hard edge model with the formula 86 and 87

In conclusion, for small perturbations, the number of betatron oscillations in the hard edge model of FFAG can be approximated by the formula 86 and 87 above.

$2^{\text {nd }}$ case: we let the scaling factors be arbitrary functions of the radius. Yet, we drop the assumption of the similarity of the closed orbits. This implies that the bending angles $\theta_{F}$ and $\theta_{D}$ are functions of the radius. This also suggests that different closed orbits might cross each other.

We have 6 variables in the system $\left(\rho_{F}, \rho_{D}, R_{F}, R_{D}, \theta_{F}, \theta_{D}\right)$ requiring a system of 5 equations in order to express the solutions as a function of $R_{F}$. From the previous results, we set the system:

$$
\left\{\begin{array}{l}
\frac{\rho_{F}}{R_{F}}=\frac{\sin \left(\frac{\alpha_{F}}{2}\right)}{\sin \left(\frac{\alpha_{F}}{2}\right)+\sin \left(\frac{\theta_{F}-\alpha_{F}}{2}\right)} \\
\frac{\rho_{D}}{R_{D}}=-\frac{\sin \left(\alpha_{D}\right)}{\cos \left(\alpha_{D}+\alpha_{B}+\frac{\theta_{D}-\theta_{F}+\alpha_{F}}{2}\right)} \times \frac{\cos \left(\frac{\theta_{D}}{2}\right)}{\sin \left(\theta_{D}\right)} \\
\frac{R_{D}}{R_{F}}=\frac{R_{D}}{L_{B}} \times \frac{L_{B}}{\rho_{F}} \times \frac{\rho_{F}}{R_{F}} \\
\frac{\rho_{F}}{\rho_{D}}=\frac{B_{D 0}}{B_{F 0}} \times \frac{R_{D}^{k_{D}}}{R_{F}^{k_{F}}} \times R_{0}^{k_{F}-k_{D}} \\
\theta_{F}-2 \theta_{D}=\frac{2 \pi}{N} \quad ; \quad \theta_{F, D} \geqslant 0
\end{array}\right.
$$

The first 3 equations result from the hard edge model. The 4th equation expresses the change of the bending radii with the particle momentum while the last one expresses the periodicity of the equilibrium orbit, with $2 \pi / N$ deflection in one sector. This system is highly non-linear, and we do not solve it here. Instead, the Bogoliubov method of averages is invoked in the next section to remediate this problem. 


\section{Method of averages}

Using the Bogoliubov-Krilov-Mitropolsky (BKM)'s method of averages [17], one can compute approximately the frequencies of betatron oscillations and their dependence on the average field index of the $\mathrm{F}$ and $\mathrm{D}$ magnets. This method is characterized by the expansion of the dependent variables in power series of a small parameter as detailed below.

We start from Eqs. (19) where the damping term is removed, and re-write the equation of motion for each closed orbit in cylindrical coordinates $(R, \theta)$ : differentiating with respect to the azimuthal angle $\theta$, one obtains:

$$
\begin{aligned}
\frac{d x}{d \theta} & =\frac{d x}{d s} \frac{d s}{d \theta} \\
\frac{d^{2} x}{d \theta^{2}} & =\frac{d^{2} x}{d s^{2}}\left(\frac{d s}{d \theta}\right)^{2}+\frac{d x}{d s} \frac{d^{2} s}{d \theta^{2}}
\end{aligned}
$$

Given that the longitudinal position $\mathrm{s}$ is quasi-proportional to the azimuthal angle, one can neglect the $2^{\text {nd }}$ term derivative $\left(d^{2} s / d \theta^{2} \approx 0\right)$. Also, the arclength in cylindrical coordinates is given by:

$$
d s^{2}=d R^{2}+R^{2} d \theta^{2}+d z^{2}
$$

Thus, assuming that the motion is restricted to the median plane, this yields:

$$
\frac{d s}{d \theta}=\sqrt{R^{2}+\left(\frac{d R}{d \theta}\right)^{2}} \approx R
$$

so that the equations of motion write:

$$
\left\{\begin{array}{l}
\frac{d^{2} x}{d \theta^{2}}+\left[\mu^{2}(R, \theta)[1-n]\right] x=0 \quad ; \quad \mu(R, \theta)=\frac{R}{\rho} \\
\frac{d^{2} y}{d \theta^{2}}+\left[\mu^{2}(R, \theta) n\right] y=0
\end{array}\right.
$$

Now, Eqs. (93) can be written in cylindrical coordinates, in the standard form:

$$
\frac{d^{2} x}{d \theta^{2}}+g(R, \theta) x=0
$$

where $\mathrm{R}$ is a parameter that refers to the local radius of the closed orbit at a given azimuth $\theta$ and $g(R, \theta)$ is periodic in $\theta$ with period $2 \pi / N$. In order to apply the averaging method, we introduce the two dimensional vector $\mathbf{X}=\left(\begin{array}{c}x \\ d x / d \Theta\end{array}\right)$ as well as the independent variable $\Theta=N \theta$. Then, the above equation can be written in the matrix form:

$$
\frac{d \mathbf{X}}{d \Theta}=\frac{1}{N} \mathbf{G X}
$$

where

$$
\mathbf{G}=\left(\begin{array}{cc}
0 & 1 \\
-g(R, \Theta) & 0
\end{array}\right)
$$

The main idea of the averaging method is based on the assumption that the derivative $d \mathbf{X} / d \Theta$ is small. Therefore, one can represent $\mathbf{X}$ as the sum of two terms, a slowly varying term $\xi$, and small rapidly oscillating terms. If $\mathrm{N}$ is large ( $\mathrm{N}$ $=12$ in our case), this is generally the case and a solution of (95) can be obtained in the form of series of the powers of $1 / \mathrm{N}[18]$ :

$$
\mathbf{X}(R, \Theta)=\left[1+\frac{1}{N} \tilde{\boldsymbol{G}}+\frac{1}{N^{2}}(\tilde{\boldsymbol{G} \tilde{\boldsymbol{G}}}-\tilde{\tilde{\boldsymbol{G}}}\langle\boldsymbol{G}\rangle)+\ldots\right] \xi
$$

where $\sim$ is the tilde operator defined by:

$$
\tilde{\mathbf{G}}(R, \Theta)=\int[G(R, \Theta)-\langle G(R, \Theta)\rangle] d \Theta
$$

and \langle\rangle is the average operator defined by:

$$
\langle\mathbf{G}(R, \Theta)\rangle=\lim _{T \rightarrow \infty} \frac{1}{T} \int_{0}^{T} \mathbf{G}(R, \Theta) d \Theta
$$

Now, a solution of Eq. (97) is described by the formula

$$
x=A e^{i \nu \theta}\left[1-\frac{1}{N^{2}} \tilde{\tilde{g}}+\ldots\right]
$$


where $\nu$ is an approximate solution of the number of betratron oscillations per turn and is given by:

$$
\begin{aligned}
\nu^{2}(R) & =\langle g\rangle+\frac{1}{N^{2}}\left\langle\tilde{g}^{2}\right\rangle+\ldots \\
& \approx\langle g(R, \theta)\rangle+\left\langle\left[\int\{g(R, \theta)-\langle g(R, \theta)\rangle\} d \theta\right]^{2}\right\rangle \\
& =g_{1}(R)+g_{2}(R)
\end{aligned}
$$

From the geometry of the equilibrium orbit and the approximation made in Eq. (92), it can be shown that:

$$
\begin{aligned}
& \frac{d s}{d \theta}=R \cos (\phi) \approx R \\
& \frac{\partial \theta}{\partial x}=\frac{\sin (\phi)}{R} \approx 0 \\
& \frac{\partial R}{\partial x}=\cos (\phi) \approx 1
\end{aligned}
$$

Now, we calculate the field index n:

$$
n=-\frac{\rho}{B} \frac{\partial B}{\partial x}=-\frac{\rho}{B}\left[\frac{\partial B}{\partial R} \frac{\partial R}{\partial x}+\frac{\partial B}{\partial \theta} \frac{\partial \theta}{\partial x}\right] \approx-\frac{\rho}{R} k(R)
$$

Now, back to Eqs. (41). The preceding formalism applies by simply replacing $g(R, \theta)$ by [24]:

$$
\left\{\begin{array}{l}
g_{x}(R, \theta)=\left(\frac{R}{\rho}\right)^{2} \times(1-n) \approx\left(\frac{R}{\rho}\right)^{2} \times\left[1+\frac{\rho}{R} k(R)\right] \\
g_{y}(R, \theta)=\left(\frac{R}{\rho}\right)^{2} \times n \approx-\frac{R}{\rho} \times k(R)
\end{array}\right.
$$

We calculate each term in Eq. (101) separately by introducing the azimuthal variation of the scaling factor in the following way:

$$
k(R, \theta)= \begin{cases}k_{F}(R), & \text { if } \theta \in \theta_{F} \\ k_{D}(R), & \text { if } \theta \in \theta_{D} \\ k_{\text {drift }}(R), & \text { if } \theta \in \theta_{\text {drift }}\end{cases}
$$

We start from the vertical plane and calculate the first order term which is the mean value of the forcing term in Eq. (94):

$$
\begin{aligned}
g_{1 y}\left(R_{E}\right) & =\left\langle g_{y}(R, \theta)\right\rangle \\
& =\frac{-1}{2 \pi / N} \int_{0}^{2 \pi / N} \frac{R}{\rho} \times k(R, \theta) d \theta \\
& =\frac{-1}{2 \pi / N}\left[\left(\int_{\theta_{F}} \mu(R, \theta) d \theta\right) k_{F}\left(R_{E}\right)+\left(\int_{\theta_{D}} \mu(R, \theta) d \theta\right) k_{D}\left(R_{E}\right)+\left(\int_{\theta_{\text {drift }}} \mu(R, \theta) d \theta\right) k_{d r i f t}\left(R_{E}\right)\right] \\
& =\sum_{i} \alpha_{i}\left(R_{E}\right) \times k_{i}\left(R_{E}\right) \quad ; \quad i=F, D, \text { drift }
\end{aligned}
$$

where $R_{E}$ is a parameter referring to the radius of the closed orbit of energy $\mathrm{E}$ and $\alpha_{i}\left(R_{E}\right)$ is what we will refer to in the future as "the $1^{\text {st }}$ order index of similarity of the closed orbits":

$$
\alpha_{i}\left(R_{E}\right)=\frac{-1}{2 \pi / N} \int_{\theta_{i}} \mu(R, \theta) d \theta=\frac{-1}{2 \pi / N} \int_{\theta_{i}} \frac{R}{\rho} d \theta
$$

The assumption on the similarity of the closed orbits is withdrawn here given that $\alpha_{i}$ is allowed to change with the radius. Also, note that for the $\mathrm{F}$ magnet, $R / \rho>0$, so that $\alpha_{F}<0$. However, for the $\mathrm{D}$ magnet, $\alpha_{D}>0$. Therefore, by means of Eq. (106), one can predict that, to the first order in the method of averages, the D magnet increases the vertical focusing, while the F magnet plays the opposite role. Also, the drift is included in this formula. Since the drift space between the magnets is susceptible to contain the fringe fields, it may be important in some cases to assign a scaling factor to it to determine its effect on the beam dynamics. However, in the ideal case, $\rho_{\text {drift }} \rightarrow \infty$ so that $\alpha_{d r i f t} \approx 0$.

The second order term can be calculated using:

$$
\begin{aligned}
g_{2 y}\left(R_{E}\right) & =\left\langle\left[\int\left\{g_{y}(R, \theta)-\left\langle g_{y}(R, \theta)\right\rangle\right\} d \theta\right]^{2}\right\rangle \\
& =\left\langle\left[\int\left\{g_{y}(R, \theta)-g_{1 y}\left(R_{E}\right)\right\} d \theta\right]^{2}\right\rangle
\end{aligned}
$$


The azimuthal variation of the field yields an additional positive edge focusing effect (not calculated here), so that, to the first order, the vertical tune writes:

$$
\nu_{y}^{2}\left(R_{E}\right)=\sum_{i} \alpha_{i}\left(R_{E}\right) \times k_{i}\left(R_{E}\right)+\mathcal{F}^{2}\left[1+2 \tan ^{2}(\xi)\right]
$$

where $\mathcal{F}$ is the magnetic flutter (not the flutter function $\mathrm{F}$ ) defined by:

$$
\mathcal{F}^{2}=\frac{\left\langle B^{2}\right\rangle-\langle B\rangle^{2}}{\langle B\rangle^{2}}=\frac{\left\langle F^{2}\right\rangle-\langle F\rangle^{2}}{\langle F\rangle^{2}}
$$

In general $\mathcal{F}$ is $R_{E}$-dependent.

We repeat the same steps in the horizontal plane by calculating the first order term

$$
\begin{aligned}
g_{1 x}\left(R_{E}\right)=\left\langle g_{x}(R, \theta)\right\rangle & =\frac{1}{2 \pi / N} \int_{0}^{2 \pi / N}\left[\left(\frac{R}{\rho}\right)^{2}+\frac{R}{\rho} \times k(R, \theta)\right] d \theta \\
& =\frac{1}{2 \pi / N} \int_{0}^{2 \pi / N} \mu(R, \theta)^{2} d \theta-g_{1 y}\left(R_{E}\right) \\
& =\sum_{i} \beta_{i}\left(R_{E}\right)-\sum_{i} \alpha_{i}\left(R_{E}\right) \times k_{i}\left(R_{E}\right) \quad ; \quad i=F, \text { D, drift }
\end{aligned}
$$

where $\beta_{i}\left(R_{E}\right)$ is what we will refer to in the future as "the $2^{\text {nd }}$ order index of similarity of the closed orbits":

$$
\beta_{i}\left(R_{E}\right)=\frac{1}{2 \pi / N} \int_{\theta_{i}} \mu(R, \theta)^{2} d \theta=\frac{1}{2 \pi / N} \int_{\theta_{i}}\left(\frac{R}{\rho}\right)^{2} d \theta
$$

Thus, to the first order in the method of averages, the number of betatron oscillations in the horizontal plane can be approximated by:

$$
\nu_{x}^{2}\left(R_{E}\right)=\sum_{i} \beta_{i}\left(R_{E}\right)-\sum_{i} \alpha_{i}\left(R_{E}\right) \times k_{i}\left(R_{E}\right)
$$

This result is general and applies to any type of FFRA because no assumption is made regarding the analytical form of the magnetic field except that it can be written in the general form of Eq. (64). The idea is that any magnet can be decomposed into a sum of smaller magnets for which one can attribute an average field index that is constant inside (no azimuthal dependence) yet can change with the radius. In other words, if one knows precisely the closed orbits from injection to extraction and the average field index associated to each magnet, then the number of betatron oscillations can be determined by means of the previous results.

\section{Application example: the KURRI $150 \mathrm{MeV}$ FFAG ring:}

According to the previous results, the number of betatron oscillations per turn in the KURRI DFD triplet is given, to the first order, by the following formula:

$$
\begin{aligned}
& \nu_{x}^{2}(E)=\beta_{F}(E)+2 \beta_{D}(E)+\beta_{d r i f t}(E)-\alpha_{F}(E) k_{F}(E)-2 \alpha_{D}(E) k_{D}(E)-\alpha_{d r i f t}(E) k_{d r i f t}(E) \\
& \nu_{y}^{2}(E)=\alpha_{F}(E) k_{F}(E)+2 \alpha_{D}(E) k_{D}(E)+\alpha_{d r i f t}(E) k_{d r i f t}(E)
\end{aligned}
$$

where E refers to the particle energy. We calculate the number of betatron oscillations per turn using the tracking code Zgoubi and the TOSCA field map of the DFD triplet. As was shown earlier from benchmarking, the results are in agreement with the other codes (see Fig. 2). Next, the tracking results provide the closed orbits for various particle energies which were then exploited to calculate the horizontal and vertical tunes by applying the 1st order Bogoliubov method of averages (see Eqs. (113) and (114)). The hard edge model formula that was established earlier is also tested (see Eqs. (86) and (86) ). The main difference between the Bogoliubov method of averages and the hard edge model formula is that the only parameters that may vary in the latter are the average field indices $k_{F}$ and $k_{D}$. The results are also compared to the 1st order Symon formula $\left(\nu_{x}^{2}=k+1\right.$ and $\left.\nu_{y}^{2}=-k+\mathcal{F}^{2}\right)$ in which the scaling factor $\mathrm{k}$ as well as the magnetic flutter $\mathcal{F}^{2}$ are allowed to change with the radius and were obtained from the closed orbits calculation by Zgoubi. Comparison between the different results are summarized in Figs. 17] and 18 below.

The Symon formula is less in agreement with the benchmarking results than the other calculations. There are two reasons for such a disagreement: first, the Symon model assumes that the scaling factor is constant and is azimuthally invariant. Such an assumption is withdrawn in our calculation given that we define an average field index for each magnet (azimuthal variation) that changes with the radius as well. This gives rise to an orbit distortion that is accounted for by the first and second order index of similarity of the closed orbits. These quantities exhibit strong dependence on the radius and provide an additional focusing in the horizontal plane, which is the second reason for the discrepancy. Fig. 19 shows how the second order index of similarity $\left(\beta(E)=\beta_{F}(E)+2 \beta_{D}(E)+\beta_{\text {drift }}(E)\right)$ changes with the particle energy. 


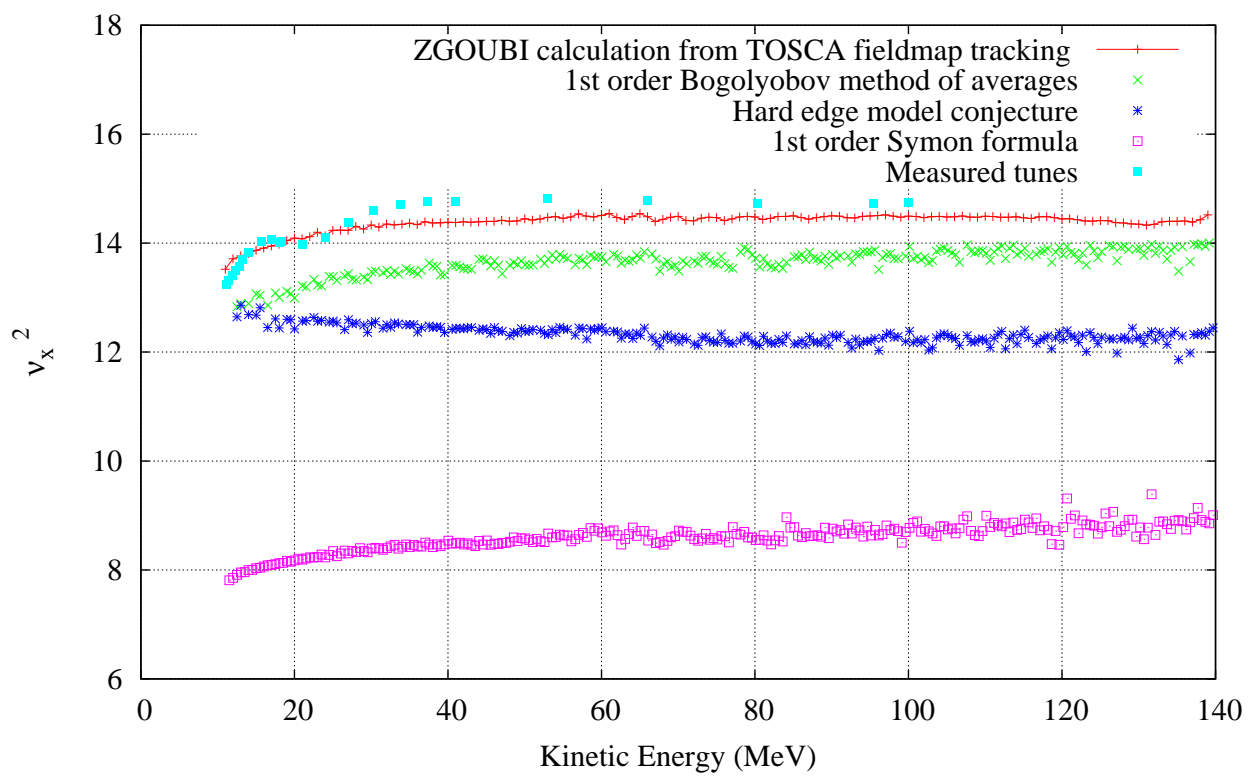

FIG. 17: Tunes calculation in the horizontal plane of the KURRI $150 \mathrm{MeV}$ FFAG ring and comparison with the analytical formula as well as the measurement.

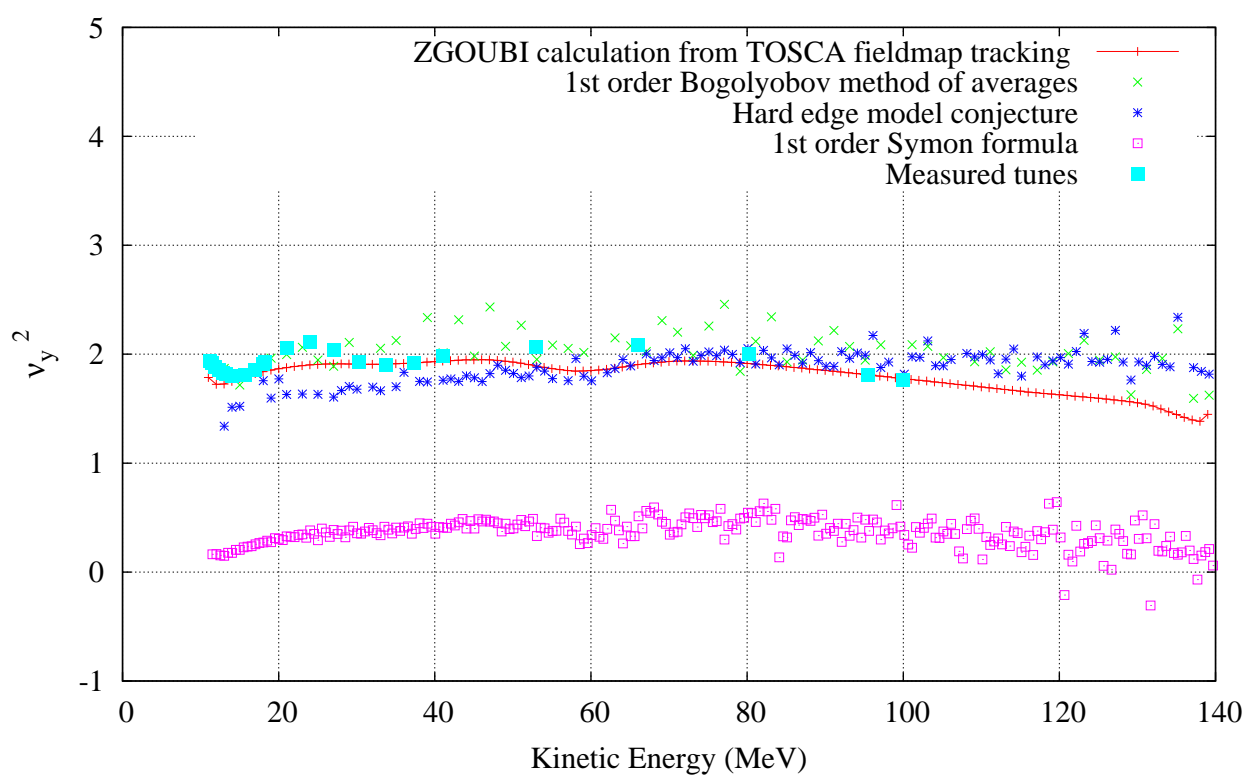

FIG. 18: Tunes calculation in the vertical plane of the KURRI $150 \mathrm{MeV}$ FFAG ring and comparison with the analytical formula as well as the measurement.

\section{ZGOUBI model}

For a scaling FFAG, an important quantity is the tune which is supposed to remain constant over the entire range of energies. From the hard edge model and the Bogoliubov method of averages, it was found that the tune is sensitive to the average field index $k_{F}$ and $k_{D}$ of the $\mathrm{F}$ and $\mathrm{D}$ magnet respectively. In other words, breaking the scaling law, although a major source of imperfection in scaling FFAG, can also be utilized in order to control the tune path in FFAG. In order to quantify the source of imperfection, we introduce two new quantities in the calculation: the average value of the tunes $\nu_{x}^{m}=\left\langle\nu_{x}\right\rangle$ and $\nu_{y}^{m}=\left\langle\nu_{y}\right\rangle$ over the closed orbits to quantify the average focusing strength of the applied forces on the beam, and the rms value of the tunes $\nu_{x}^{r m s}=\left\langle\nu_{x}^{2}\right\rangle^{1 / 2}$ and $\nu_{y}^{r m s}=\left\langle\nu_{y}^{2}\right\rangle^{1 / 2}$ to account for scaling imperfections, i.e. for any tune variations. One could use the $|\max -\min |$ value of the tunes to account for the oscillations. However, the rms quantities have the merit to be average quantities, thus more appealing to use in order to obtain smooth variations of the described quantities.

Given that the energy gain per turn is small (2 keV per turn), one can reasonably assume that the accelerated particle trajectory for a given energy is quasi the same as the closed orbit trajectory. There are two criteria to ensure the stability of the lattice: the first is to find all closed orbits between injection energy and extraction (or final) energy. In our case, we limit this study to $E_{i n j}=11 \mathrm{MeV}$ and $E_{e x t}=100 \mathrm{MeV}$, suitable for the KURRI FFAG. The second criteria 


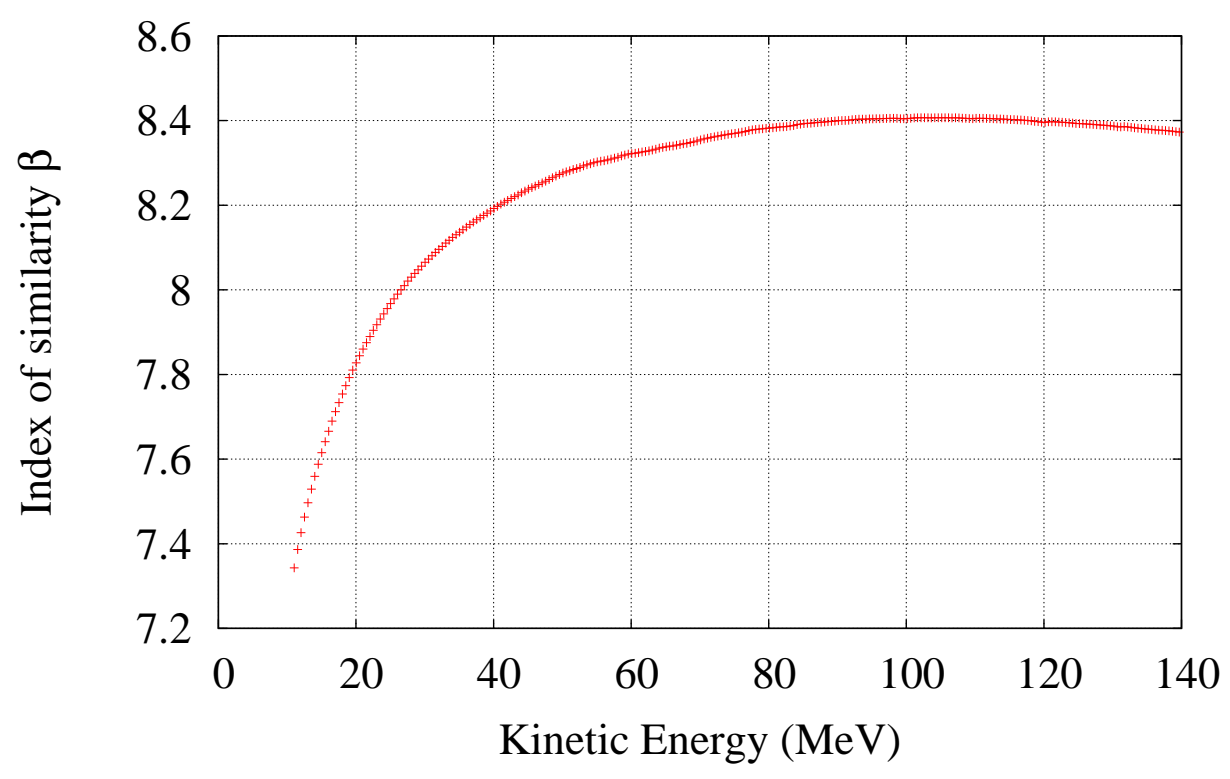

FIG. 19: $2^{\text {nd }}$ order index of similarity of the closed orbits $\beta$.

is that the number of betatron oscillations of the particles, which is obtained from multi-turn tracking around each of the closed orbits, be strictly positive. The first criteria is justified by the fact that a solution to the system of Eqs (88) does not always exist. The second criteria ensures that the motion is stable around each closed orbit.

In summary, the procedure is detailed below:

1. Generate a field map for a given $\left(k_{F}, k_{D}\right)$. The field can be written in cylindrical coordinates as:

$$
B(R, \theta)=B_{F 0} \times\left(\frac{R}{R_{0}}\right)^{k_{F}} \times F_{F}(\theta)+B_{D 0} \times\left(\frac{R}{R_{0}}\right)^{k_{D}} \times F_{D}(\theta)
$$

where $F_{F}(\theta)$ and $F_{D}(\theta)$ are the flutter functions describing the azimuthal dependence of the field and that are equal to zero in the $\mathrm{D}$ and $\mathrm{F}$ magnet, respectively. If $k_{F}=k_{D}$, the field writes in the standard form of (Eq 46).

Note that the field is separable in radial and azimuthal coordinates since the flutter functions merge to zero in the drift space between the F and D magnets as can be seen in Fig. (20).

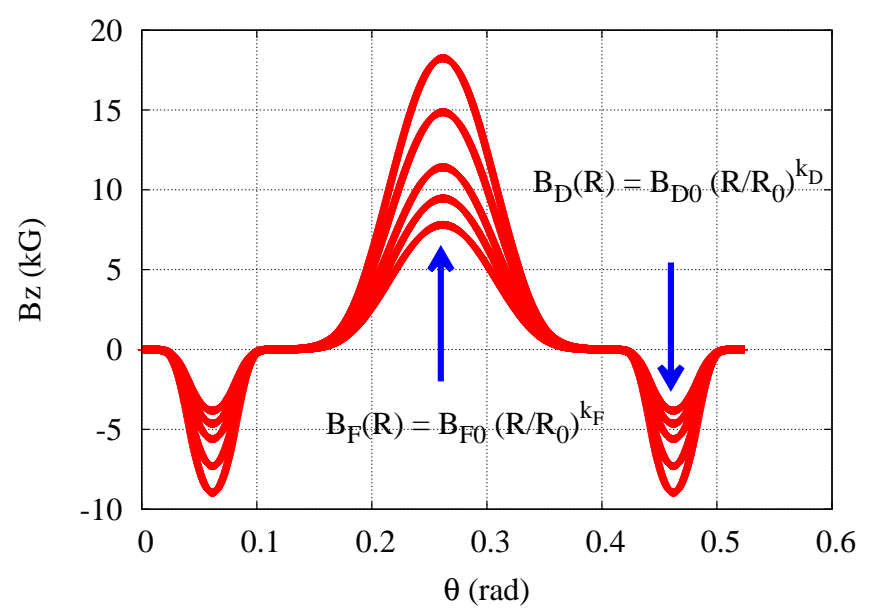

FIG. 20: Magnetic field along several closed orbits.

2. Search for NCO closed orbits between injection and extraction using the built-in fitting routines in ZGOUBI. NCO was chosen to be 30 in order to have good statistics and ensure the convergence of the calculated quantities. A typical example of 4 closed orbits search is illustrated in Fig. (21).

3. For each closed orbit, the number of betatron oscillations in both planes is computed using the ZGOUBI Discrete 


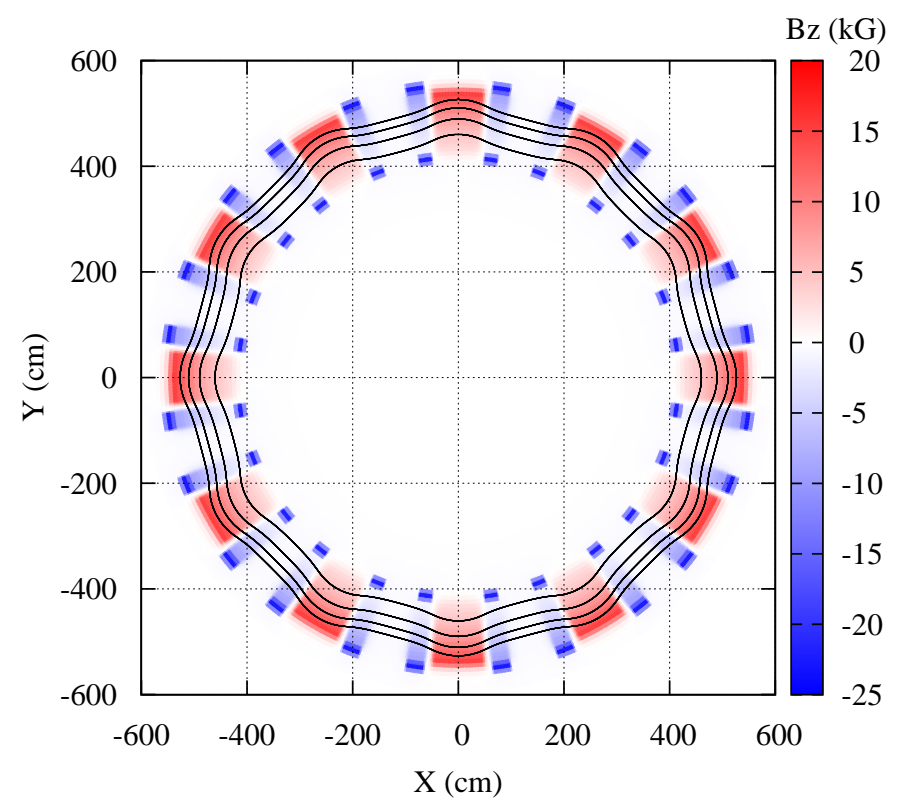

FIG. 21: Example of several closed orbits for a scaling FFAG.

Fourier Transform (DFT), from which the average as well as the rms values of the tunes are deduced:

$$
\begin{aligned}
\nu_{x, y}^{m} & =\left\langle\nu_{x, y}\right\rangle=\frac{1}{N C O} \sum_{i=1}^{N C O} \nu_{x, y, i} \\
\nu_{x, y}^{r m s} & =\left\langle\nu_{x, y}^{2}\right\rangle^{1 / 2}=\left(\frac{1}{N C O} \sum_{i=1}^{N C O}\left(\nu_{x, y, i}-\nu_{x, y}^{m}\right)^{2}\right)^{1 / 2}
\end{aligned}
$$

The merit of the DFT transform is that the assumption of the linear motion made by using Eq (75) is now withdrawn. This method simply relies on the particle coordinates stored turn after turn from particle tracking in ZGOUBI which solves the non-linear equation of motion.

The tracking code Zgoubi that we use for our analysis, solves the non-linear equation of motion [25] using truncated Taylor expansions of the field and its derivatives up to the 5 th order. Therefore it is much more accurate than the linear approach which is restrained to the first order.

Now, a scan on $k_{F}$ and $k_{D}$ provided the stability diagrams of the DFD triplet in the transverse plane (see Figs. 22 and 23 ). Qualitatively, it shows that, in the case where $k_{F}=k_{D}=k$, the average tune exhibits the right behavior predicted by the Symon formula (85): increasing $\mathrm{k}$ increases the horizontal tune and decreases the vertical one. Besides, the RMS tune exhibits the expected behavior in the vicinity of the line $k_{F}=k_{D}$ where it becomes negligible.

One can also observe that for high $\mathrm{k}$ values, any design imperfection will make the orbits quickly unstable, thus the stability diagram shrinks [26]. This is expected since the second order term in the field expansion becomes non-negligible so that the system is no longer slowly (linearly) responding to perturbations:

$$
\begin{aligned}
B(R) & =B_{0} \times\left(\frac{R}{R_{0}}\right)^{k}=B_{0} \times\left(1+\frac{x}{R_{0}}\right)^{k} \quad ; \quad x \ll R_{0} \\
& =B_{0} \times\left[1+k \frac{x}{R_{0}}+k(k-1)\left(\frac{x}{R_{0}}\right)^{2}+\ldots+\frac{k(k-1) \ldots(k-n+1)}{n !}\left(\frac{x}{R_{0}}\right)^{n}+O\left(\left(\frac{x}{R_{0}}\right)^{n+1}\right)\right]
\end{aligned}
$$

Furthermore, this study revealed some interesting results: although the rms tune variations become quite sensitive to scaling imperfections near the boundary of stability, it is observed that in the vicinity of the central line, these quantities are mostly proportional to the difference of the scaling factors as shown in Fig. (26) and can be written in the form:

$$
\nu_{x, y}^{r m s} \approx a_{x, y}\left|k_{F}-k_{D}\right|
$$

This result can also be inferred from the contour plot (see Figs. 22b and 23b). Modifying the colorbar range in Fig. $(22 \mathrm{~b})$ enabled us to clearly observe the rms tune variations in the inner region of the stability diagram (see Fig. (24)). We will exploit this result later on when we establish a way to counteract the rms tune variations introduced by the space charge effects in scaling FFAG, the idea being to perturb $k_{F}$ or $k_{D}$.

Another important result that this study revealed is that the vertical tune exhibits the same behavior vis $\grave{a}$ vis $k_{F}$ and $k_{D}$, however, for the horizontal tune, the situation is strongly related to whether $k_{D}>k_{F}$ or the opposite (see Fig. (25)). This will be discussed more in detail in the next section.

The implication of that is essentially, by implementing trim coils, similar to those that cyclotrons have, one can play with 


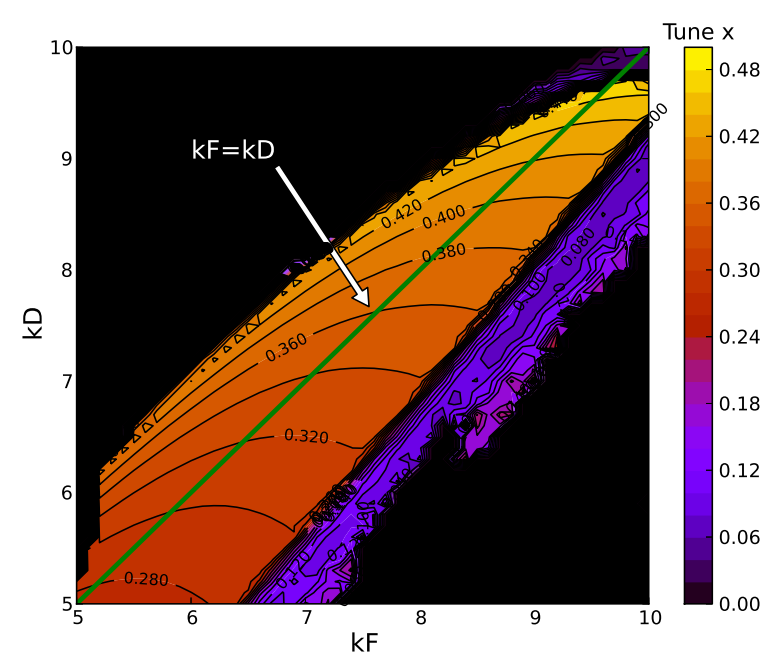

fig1Average tune of the DFD triplet in the horizontal plane.

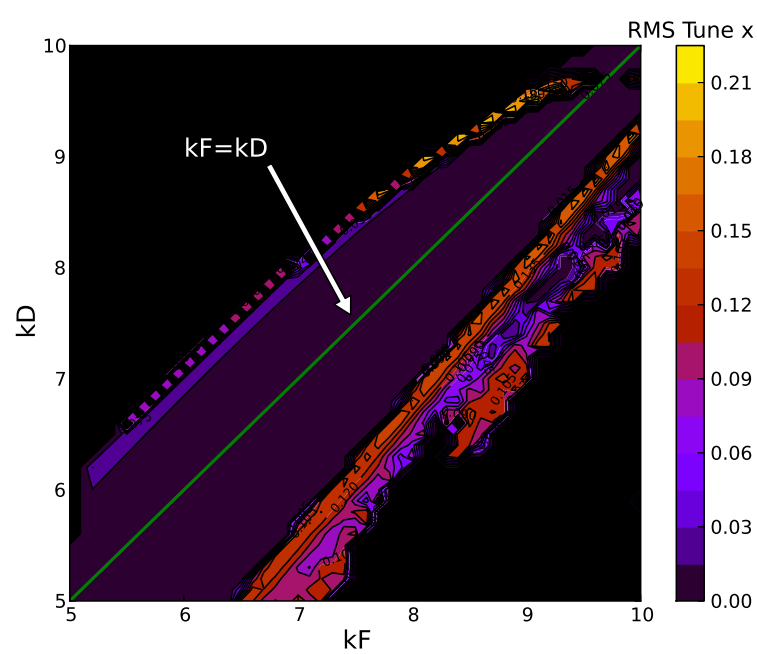

fig2RMS tune variations of the DFD triplet in the horizontal plane.

FIG. 22: Contour plot of the average and rms tune variations in the horizontal plane as a function of the scaling factor $k_{F}$ and $k_{D}$.

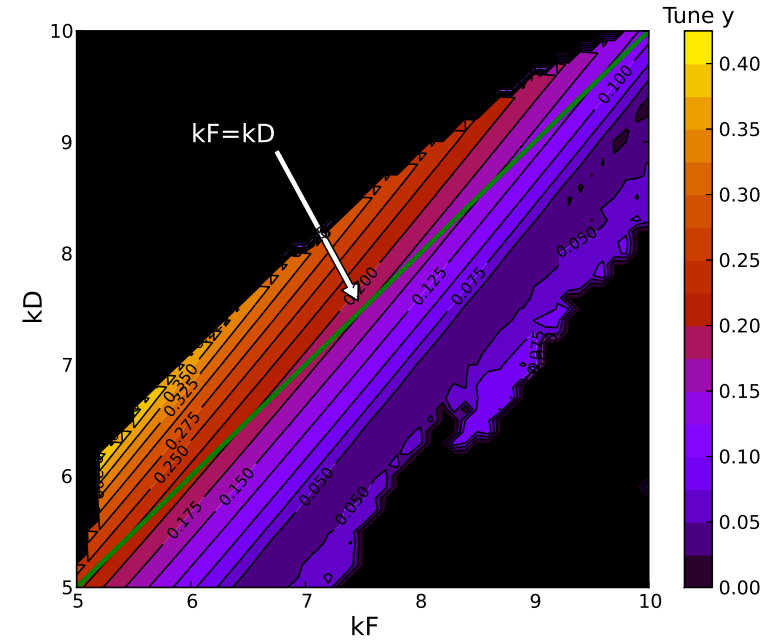

fig1Average tune of the DFD triplet in the vertical plane.

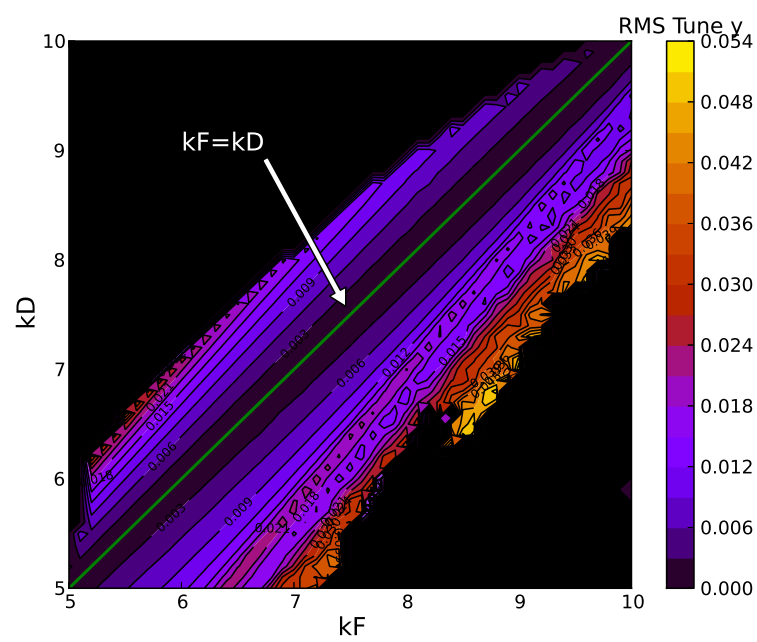

fig2RMS tune variations of the DFD triplet in the vertical plane.

FIG. 23: Contour plot of the average and rms tune variations in the vertical plane as a function of the scaling factor $k_{F}$ and $k_{D}$ : as can be observed from this plot, whenever $k_{F} \neq k_{D}$ the rms tune variations increase considerably.

the tune in a flexible way: modifying $k_{F}$ or $k_{D}$ or $k_{F}$ and $k_{D}$ at the same time, would allow to change the tune path quite in both planes. The major advantage would be to avoid crossing any resonances. This is in analogy with trim coils which improve the isochronism in cyclotrons. For high power application, the tune spread is an important issue. However, one can reasonably predict from the previous study that the scaling imperfections $\left(k_{F} \neq k_{D}\right)$ can be deliberately introduced in order to cure the space charge tune dispersion that tends to increase beam losses through resonance crossings.

Another important outcome of this study is that the space charge effects increase the rms tune spread: therefore the question that one will tackle is whether one can find a function $k_{F}(R)$ and $k_{D}(R)$ that will minimize the tune spread due to space charge forces and therefore maintain the scaling properties of the FFAG? Since the space charge has a defocusing effect on both planes, the average field index of the D-magnet seems to be the right parameter to play with since it has a focusing effect on both planes as shown by the ZGOUBI model(see Fig. (25).

\section{Discussion}

From the results of the hard edge model, it was shown that, under the assumption that the similarity of the orbits is maintained, the square of the number of betatron oscillations in both planes is, to the first order, a linear combination of the average field indices $k_{F}$ and $k_{D}$ of the focusing and defocusing magnets respectively. Later on, when this assumption was dropped, and, solving the linearized particle equations of motion by means of the Bogoliubov method of averages, it was found that this result can be generalized by defining the $1^{\text {st }}$ and $2^{\text {nd }}$ order indices of similarity of the closed orbits $\alpha_{i}$ and $\beta_{i}$ respectively. According to this method the effect of perturbing the field indices of the magnets may produce 


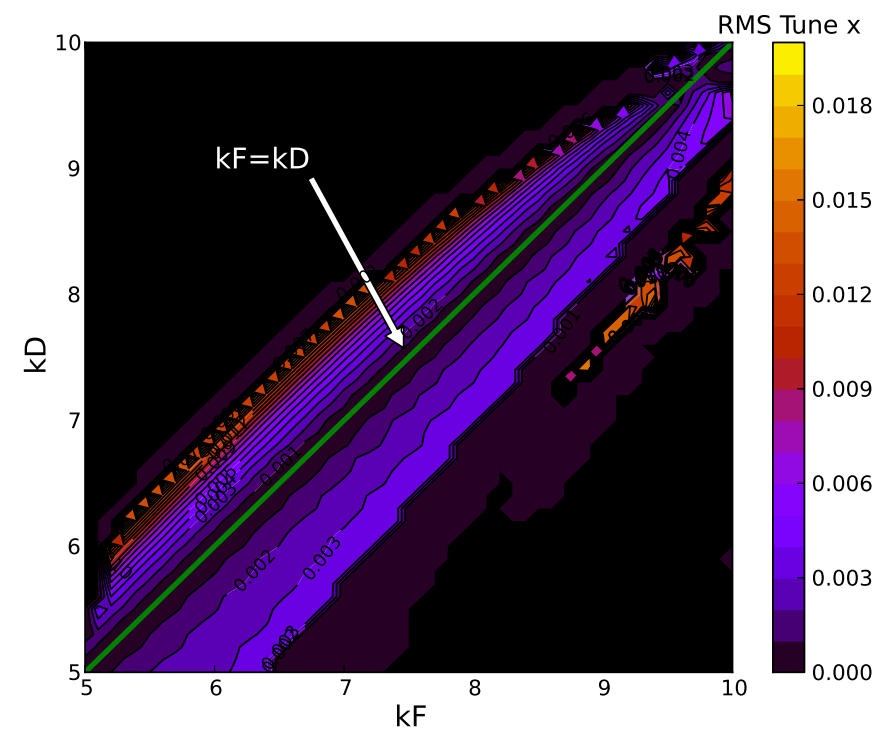

FIG. 24: RMS tune variations of the DFD triplet in the horizontal plane. (Same result as Fig. 22 b, but with a modified colorbar range).

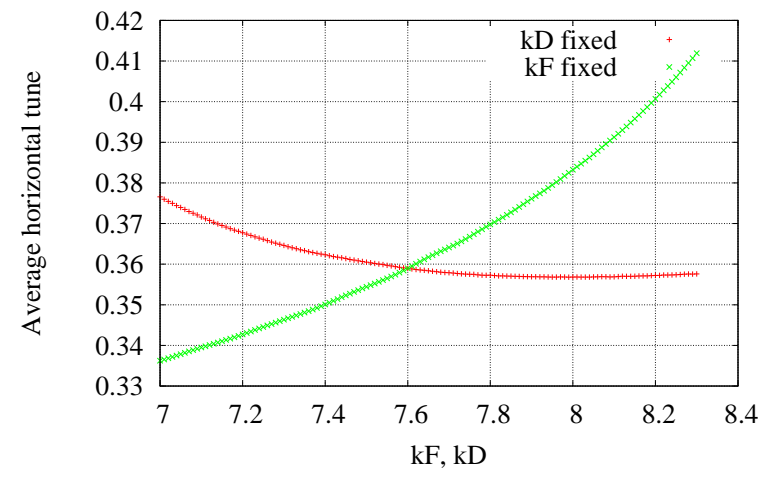

fig1Average tune of the DFD triplet in the horizontal plane.

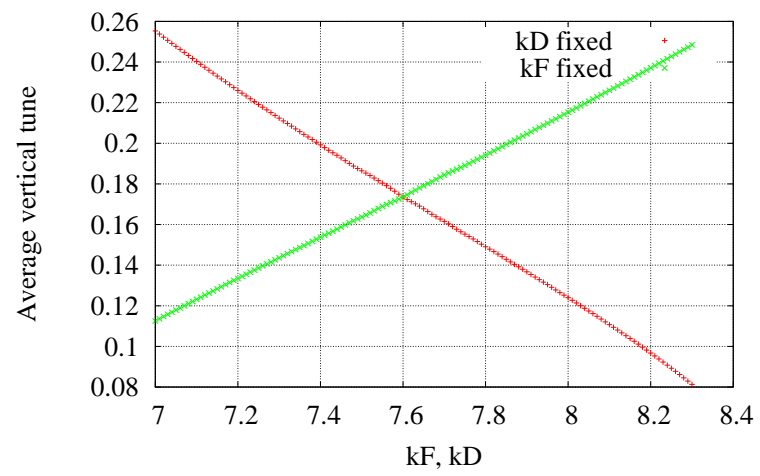

fig2Average tune of the DFD triplet in the vertical plane.

FIG. 25: Average tune variations in the transverse plane as a function of $k_{F}$ or $k_{D}$. A scan on $k_{F}$ or $k_{D}$ is performed by fixing $k_{D}$ or $k_{F}$ respectively, to the design value 7.6 .

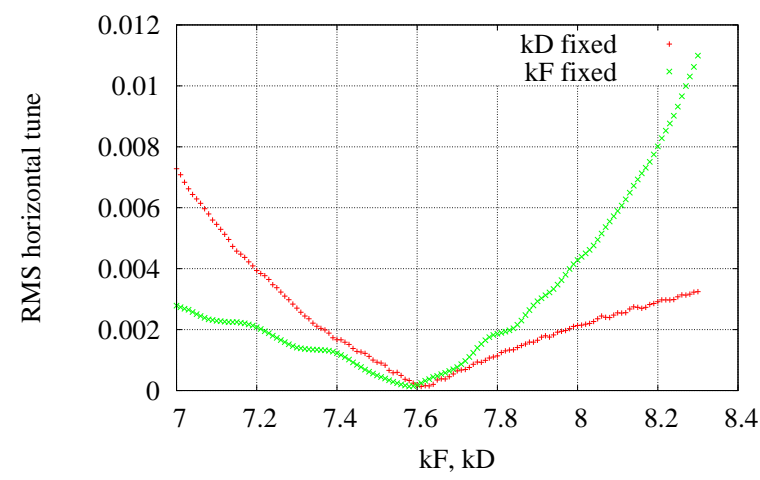

fig1RMS tune variations of the DFD triplet in the horizontal plane as a function of $k_{F}$ or $k_{D}$.

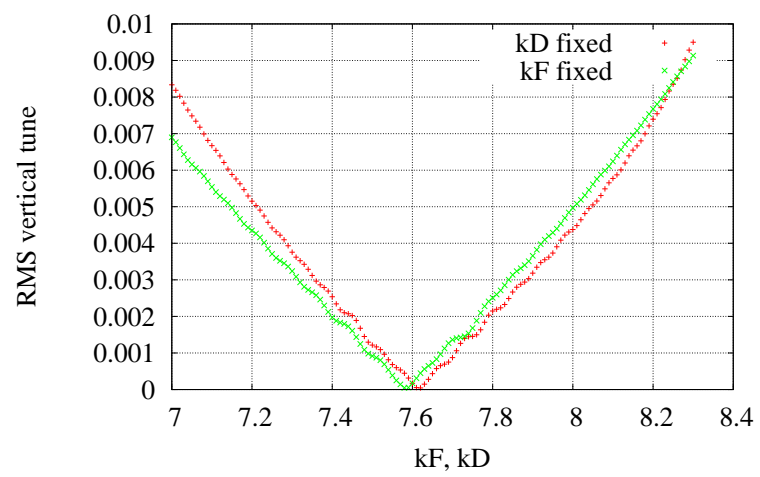

fig2RMS tune variations of the DFD triplet in the vertical plane as a function of $k_{F}$ or $k_{D}$..

FIG. 26: RMS tune variations in the transverse plane as a function of $k_{F}$ or $k_{D}$.

an orbit distortion that manifests through a radial dependence of the index of similarity of the orbits that adds to the radial dependence of the scaling factors. This changes the tune path in both planes. Besides, the magnetic flutter is susceptible to change when the scaling law is perturbed. Therefore, in this section we compare the results of solving the non-linear equation of motion using the tracking code Zgoubi with those predicted by the Bogoliubov analytical calculation based on the linearized particle equations of motion.

In what follows we exploit the results of the Zgoubi model based on the procedure that was detailed in the previous section (see Eq. (115)). The first test that was carried out to characterize the similarity of the orbits is to calculate 
the $1^{\text {st }}$ order index of similarity of the orbits averaged over the width of the DFD triplet. As can be seen in Fig. 27, if $k_{F}=k_{D}$, then the index of similarity is energy-independent. This is valid within the focusing and defocusing magnet as well (see Fig. 28) so that the second cardinal condition in Eq. (43) is satisfied. We will refer to the case where all parameters x scale, i.e $k_{F}=k_{D}=k^{r e f}$ as $x^{r e f}$. Now, we introduce a perturbation of the average field indices of the magnets by perturbing $k_{D}$ and maintaining $k_{F}$ to a fixed value. Two regimes are observed:

-If $k_{D}<k_{F}$ (down-pointing triangle in the figures) then: $\alpha_{F}<\alpha_{F}^{r e f}$ and $\alpha_{D}>\alpha_{D}^{r e f}$. Besides $\alpha_{F}(E)$ is strictly decreasing while $\alpha_{D}(E)$ is strictly increasing.

-If $k_{D}>k_{F}$ (upward-pointing triangle in the figures) then: $\alpha_{F}>\alpha_{F}^{r e f}$ and $\alpha_{D}<\alpha_{D}^{r e f}$. Besides $\alpha_{F}(E)$ is strictly increasing while $\alpha_{D}(E)$ is strictly decreasing.

Thus, both magnets act in opposition vis-à-vis scaling imperfections. This result is qualitatively in agreement with the hard edge model: solving the system of equations (88) in an iterative way yields:

$$
\text { If } \alpha_{F}(E)=\left\langle\frac{R}{\rho}\right\rangle_{F} \nearrow \Longrightarrow \theta_{F} \nearrow \Longrightarrow \theta_{D} \nearrow \Longrightarrow \alpha_{D}(E)=\left\langle\frac{R}{\rho}\right\rangle_{D} \searrow
$$

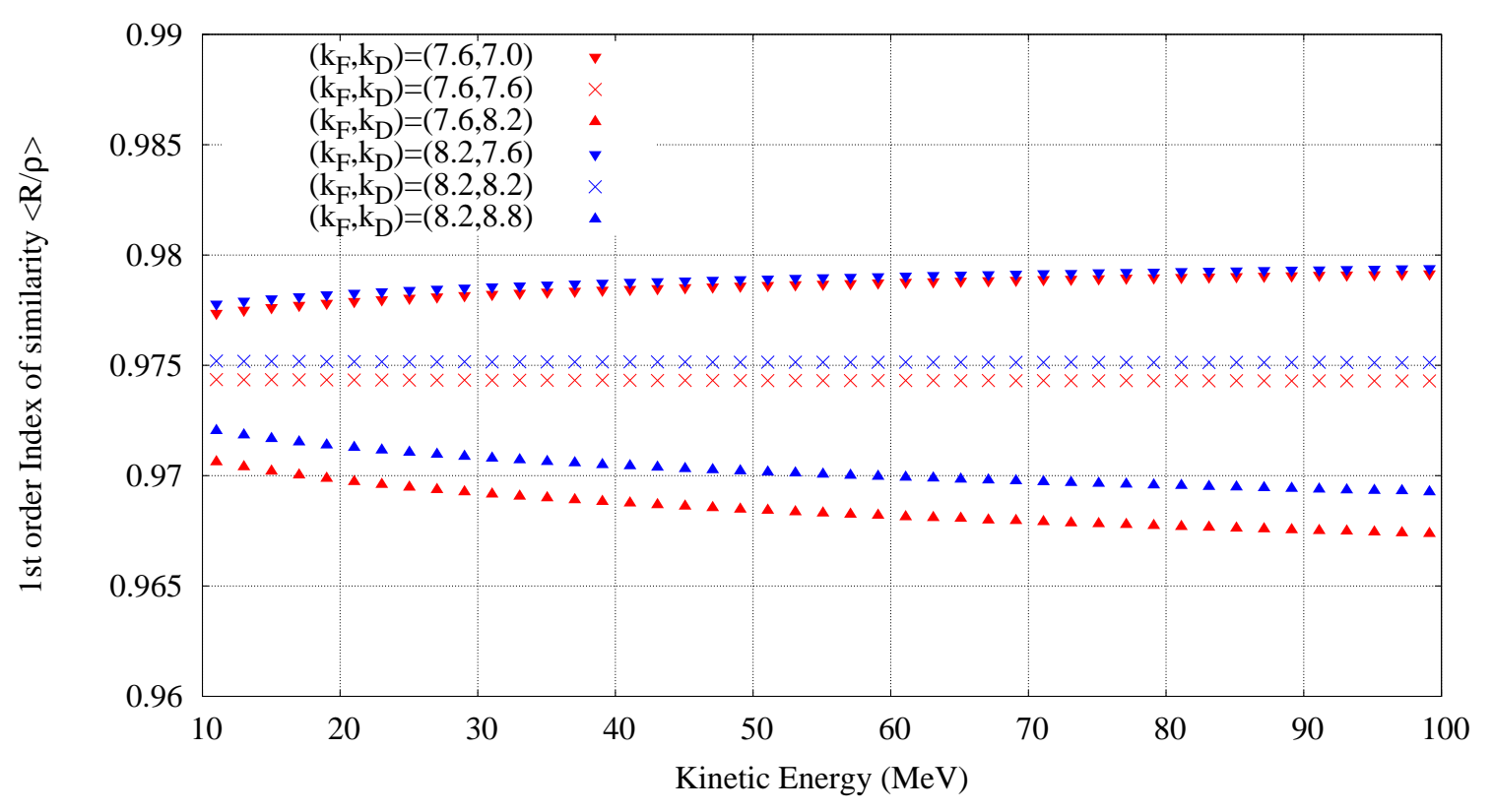

FIG. 27: $1^{\text {st }}$ order index of similarity of the closed orbits.

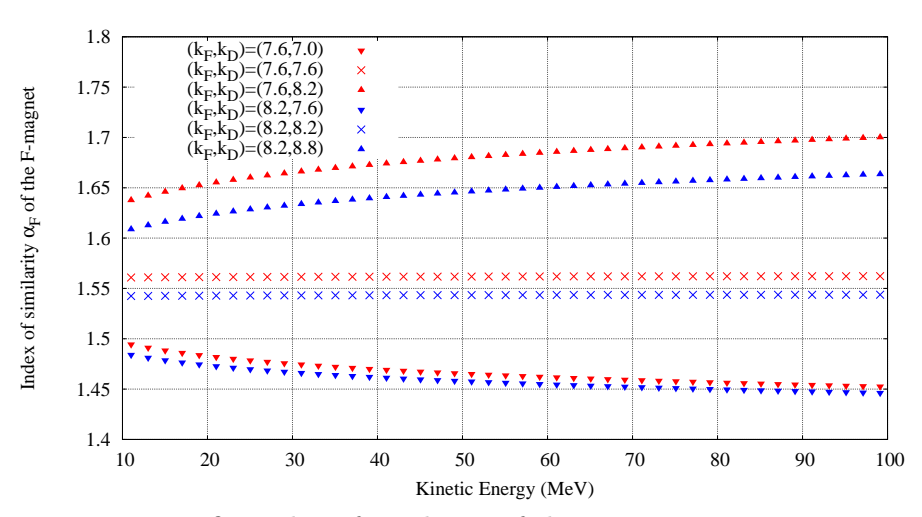

fig1Index of similarity of the F-magnet.

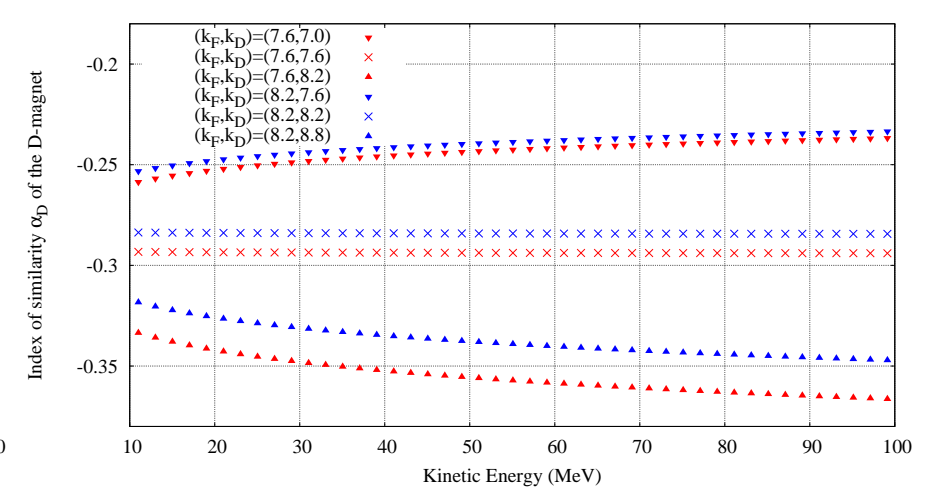

fig2Index of similarity of the D-magnet.

FIG. 28: $1^{\text {st }}$ order index of similarity of the focusing and defocusing magnets.

The $2^{\text {nd }}$ order index of similarity of the orbits as calculated from the closed orbits is shown in Fig. 30. The trend is similar to the $\alpha_{F}$ term as expected: $\langle R / \rho\rangle_{F} \gg\langle R / \rho\rangle_{D} \Longrightarrow \beta=\langle R / \rho\rangle^{2} \approx\langle R / \rho\rangle_{F}^{2}$. The $\beta$ term is particularly important as it represents the additional focusing provided by the horizontal restoring force. In order to better understand the importance of this term and the differences between the scaling and the non-scaling FFAG case, we compare the particle orbits for two samples (see Fig. 29): for the scaling FFAG case, the orbits are parallel to each other (their distance at various azimuthal positions does not change $(\delta R=$ const $)$ ). However, for the non-scaling FFAG case $\left(k_{F} \neq k_{D}\right)$, the distance increase between the orbits is essentially dominant at the centre of the F-magnet and grows with the radius. This justifies the importance of the indices $\alpha_{i}$ and $\beta_{i}$ to characterize the particle orbit. 


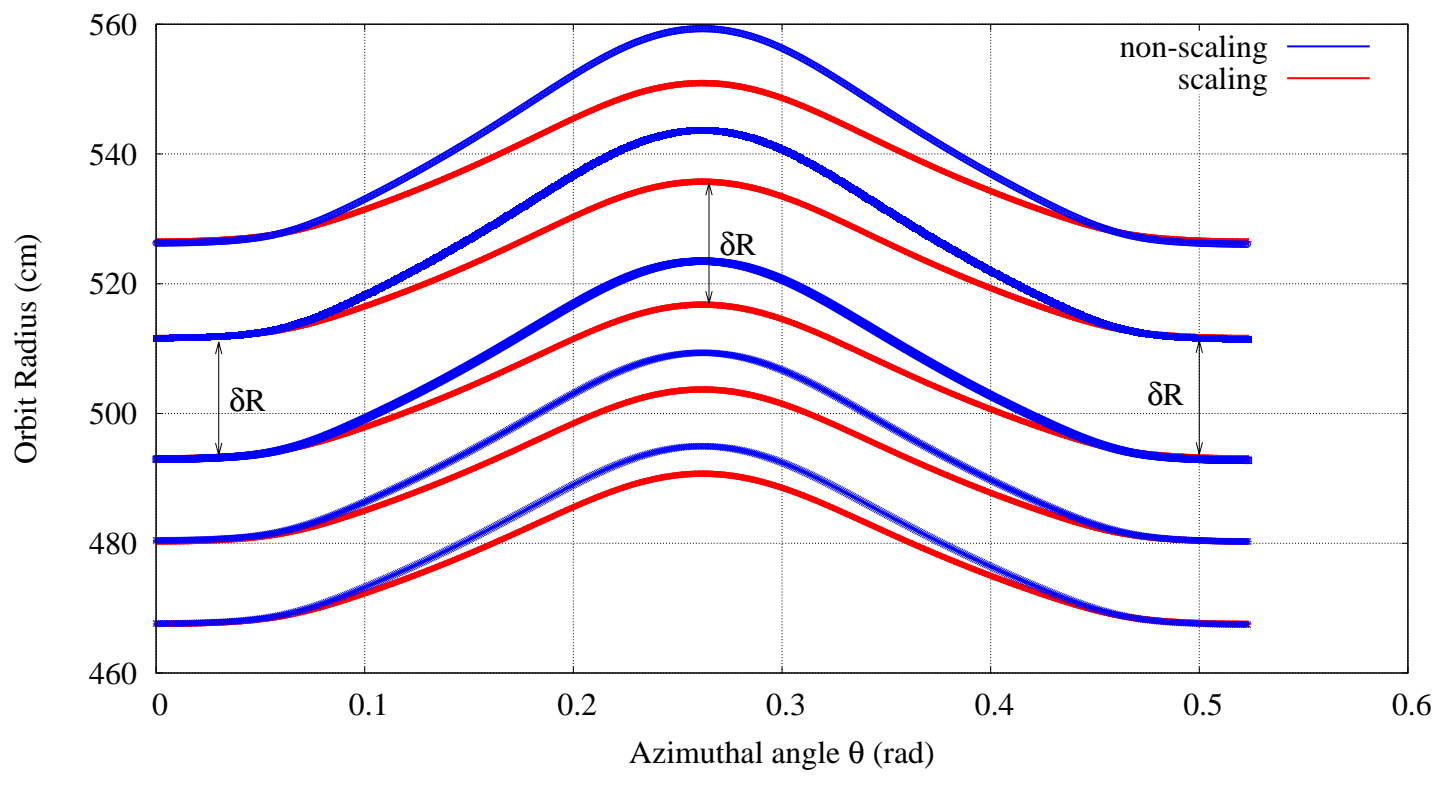

FIG. 29: Closed orbits patterns for two cases: scaling (red) vs non-scaling FFAG (blue) in which $k_{F} \neq k_{D}$.

Next, the magnetic flutter (Fig. 32) as well as the average scaling factor (Fig. 31) of the DFD triplet were computed. It is important to notice that the average scaling factor remains nearly constant $(\Delta k / k<1 \%)$ when scaling imperfections are introduced $\left(k_{F} \neq k_{D}\right)$ so that the Symon formula does not apply to explain the origin of the RMS tune variations. This also shows that having an average scaling factor $\mathrm{k}$ constant is not a sufficient condition to obtain a fixed tune machine.

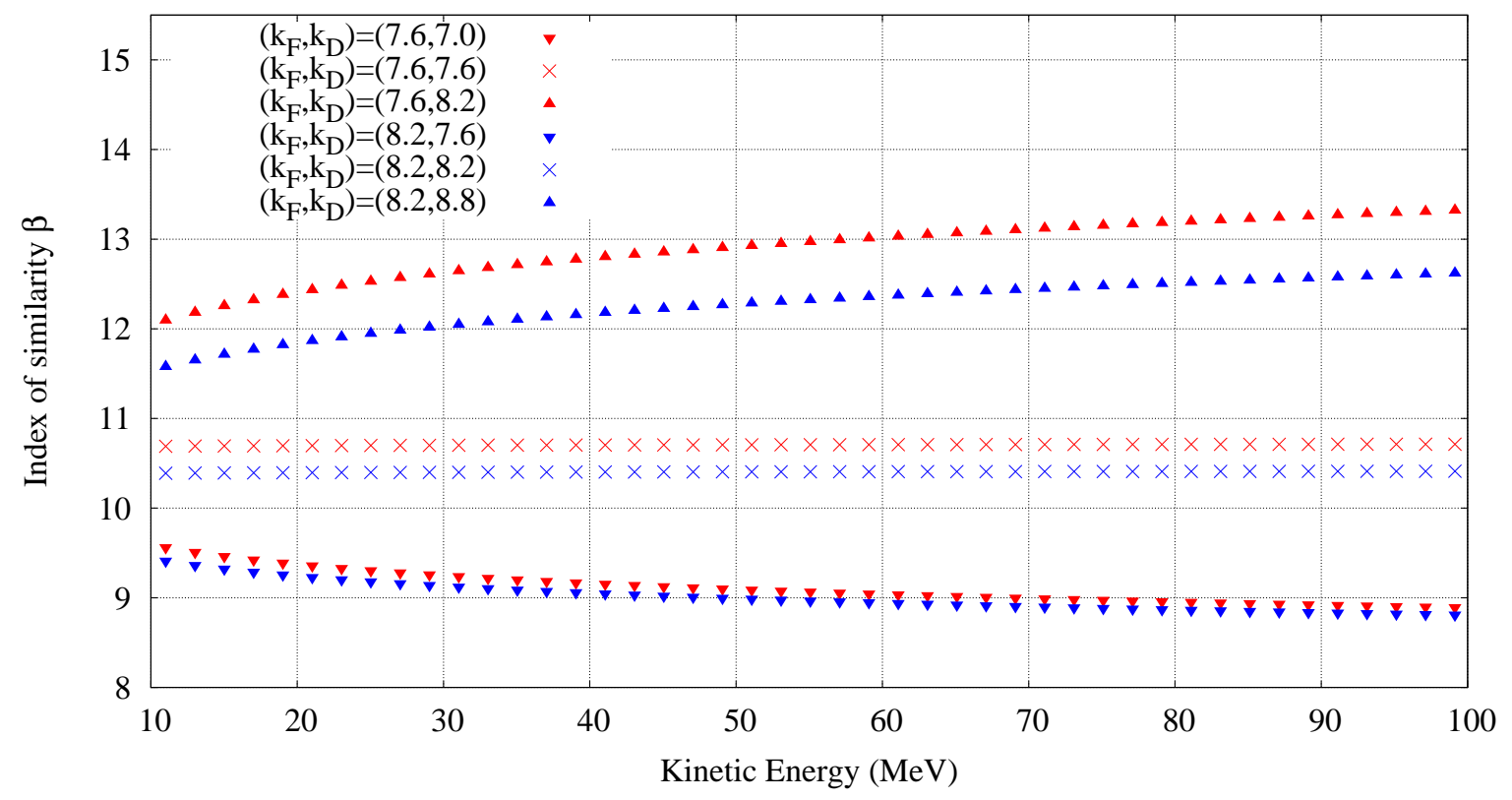

FIG. 30: $2^{\text {nd }}$ order index of similarity of the closed orbits $\beta$.

Based on all the above calculation, we compute the tunes using the Bogoliubov method of averages and compare its results with the Zgoubi calculation which is based on DFT. The results in both planes are shown in Figs. 33 and 34 both results exhibit the same behavior with the two methods and the agreement is quite good except near the boundary of stability.

Also it is observed that the tune variations in the vertical plane are mostly due to the variations of the magnetic flutter. However, in the horizontal plane, the index of similarity $\beta$ carries most of the variations.

All the above parameters characterize the orbits distortion in a scaling FFAG.

Advanced scaling FFAG concept/correction scheme?: It is important to realize that systematic errors of the magnetic field, which increase the rms tune variations quite in both planes, are unavoidable. However, based on the above results, it is possible to counteract these errors by producing an antagonistic error every two magnets in the 


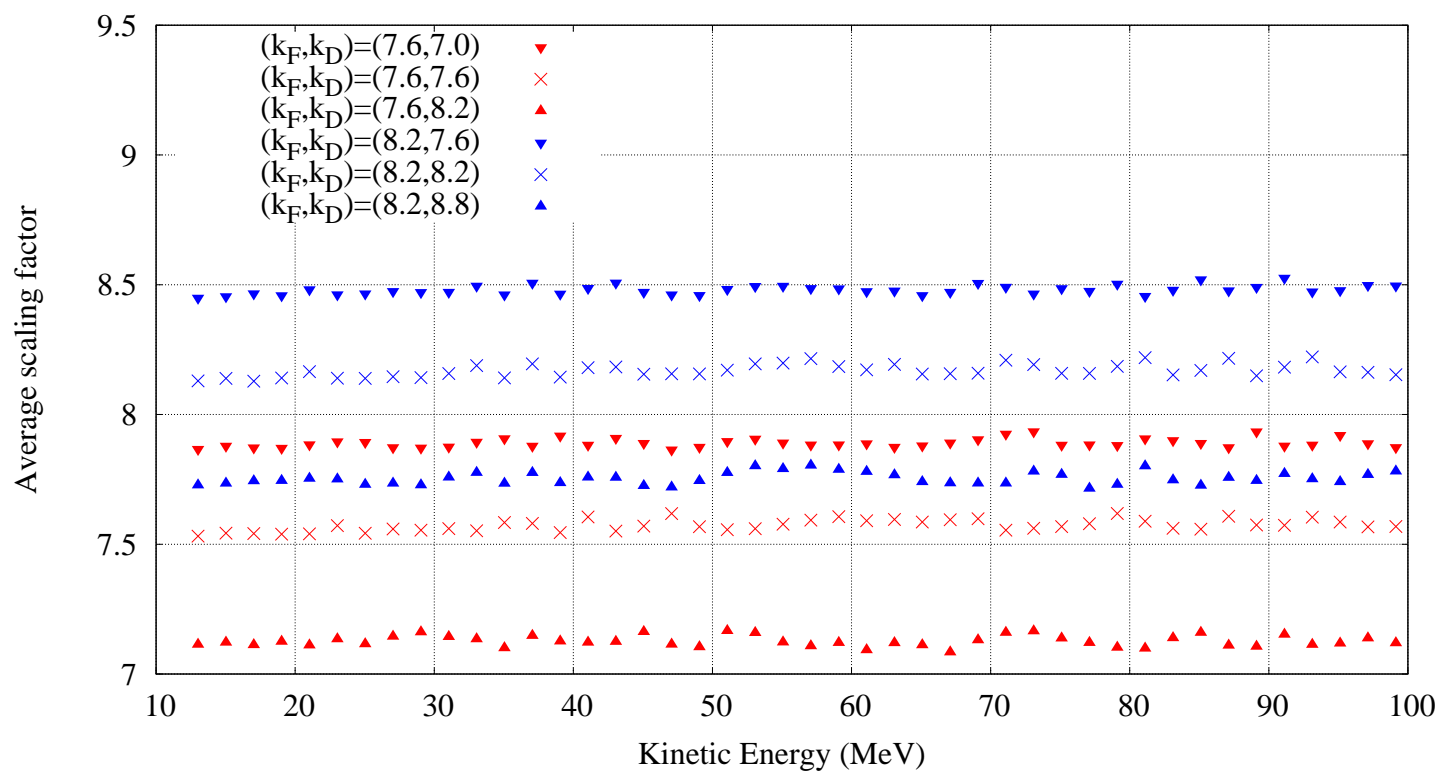

FIG. 31: $2^{\text {nd }}$ order index of similarity of the closed orbits $\beta$.

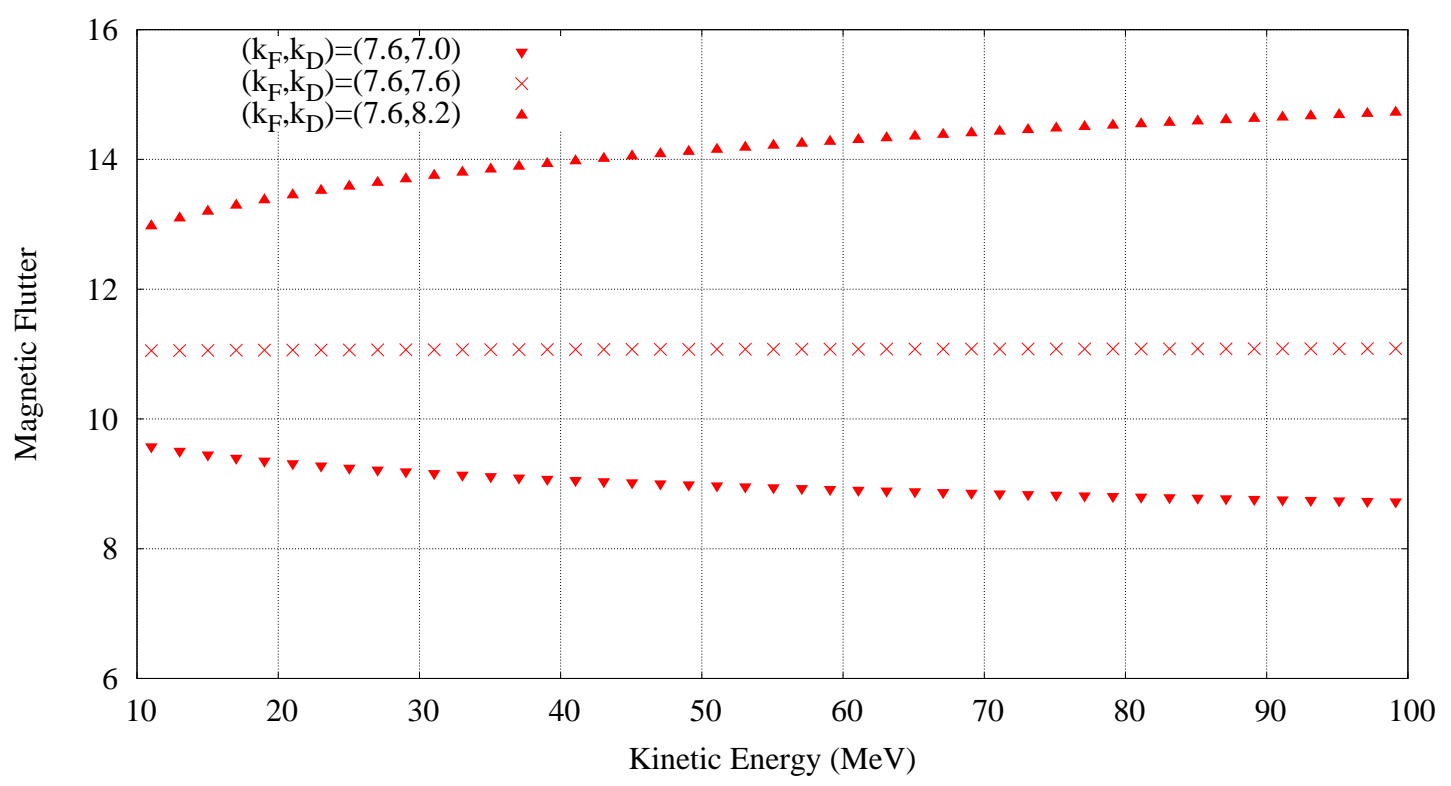

FIG. 32: Magnetic flutter $\mathcal{F}^{2}$ as a function of the kinetic energy.

following way: if $k_{F}^{s y s}>k_{D}^{\text {sys }}$, then $k_{F}^{a n t}<k_{D}^{a n t}$. More information will be provided later on.

\section{TURN SEPARATION IN A SCALING FFAG:}

In this section, we will derive the formula of the turn separation as a function of the energy, which will be useful for later analysis. We start from the scaling law:

$$
r_{2}=r_{1}\left(\frac{p_{2}}{p_{1}}\right)^{\frac{1}{1+k}}
$$

which is equivalent to:

$$
\ln \left(\frac{r_{2}}{r_{1}}\right)=\frac{1}{1+k} \ln \left(\frac{p_{2}}{p_{1}}\right)
$$

Injecting,

$$
p c=\beta \gamma m_{0} c^{2}=\left(1-\frac{1}{\gamma^{2}}\right)^{1 / 2} \gamma m_{0} c^{2}=\left(\gamma^{2}-1\right)^{1 / 2} m_{0} c^{2}
$$




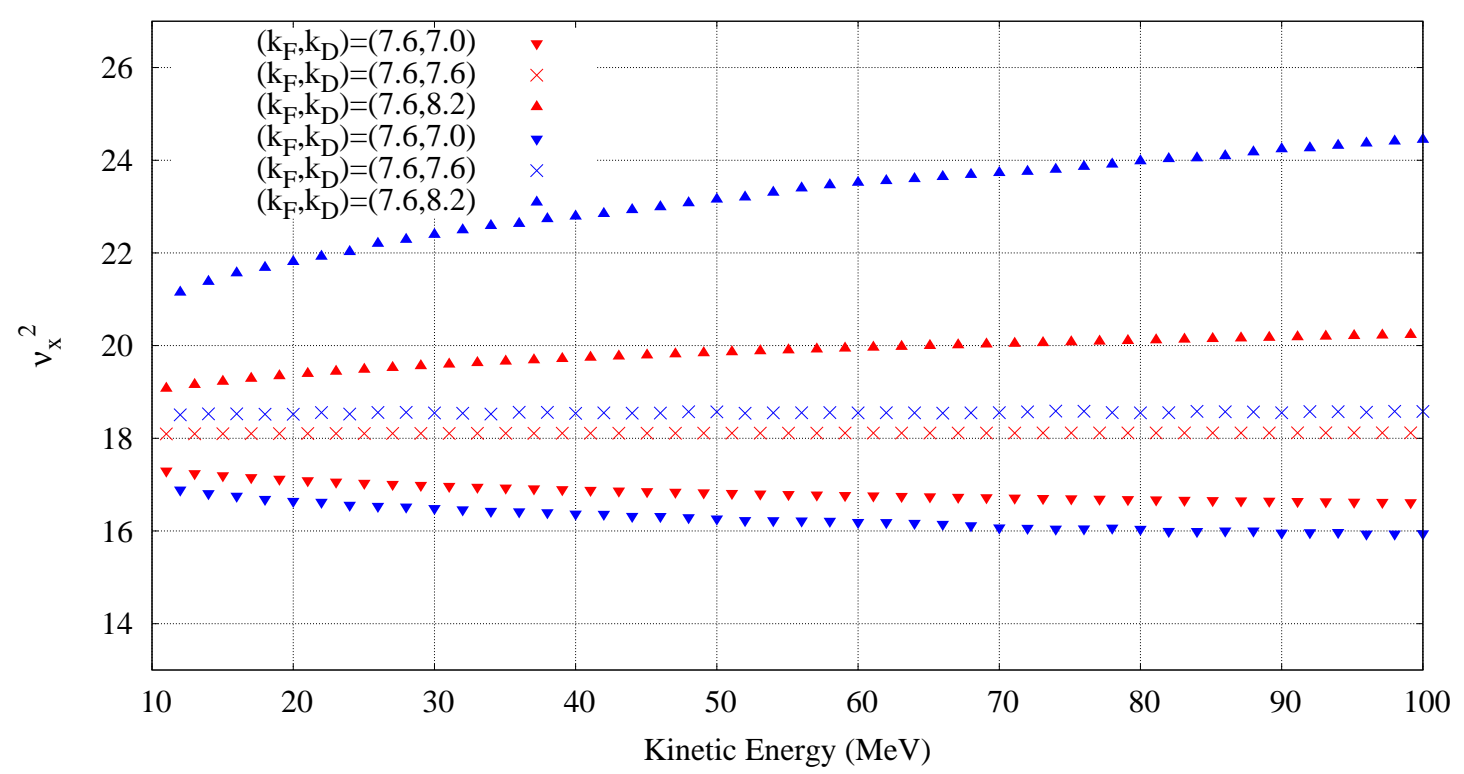

FIG. 33: Example of tune calculation using the tracking code ZGOUBI (blue) and comparison with the 1st order analytical formula 112 (red). To the first order in the method of averages, good agreement $(<4 \%)$ is obtained in the cases $\left(k_{F}, k_{D}\right)=(7.6,7.0)$ and $\left(k_{F}, k_{D}\right)=(7.6,7.6)$. However, for the last case, i.e $\left(k_{F}, k_{D}\right)=(7.6,8.2)$ the large discrepancy observed $(\lesssim 16 \%)$ is due to the fact that the beam is located nearby the boundary of stability.

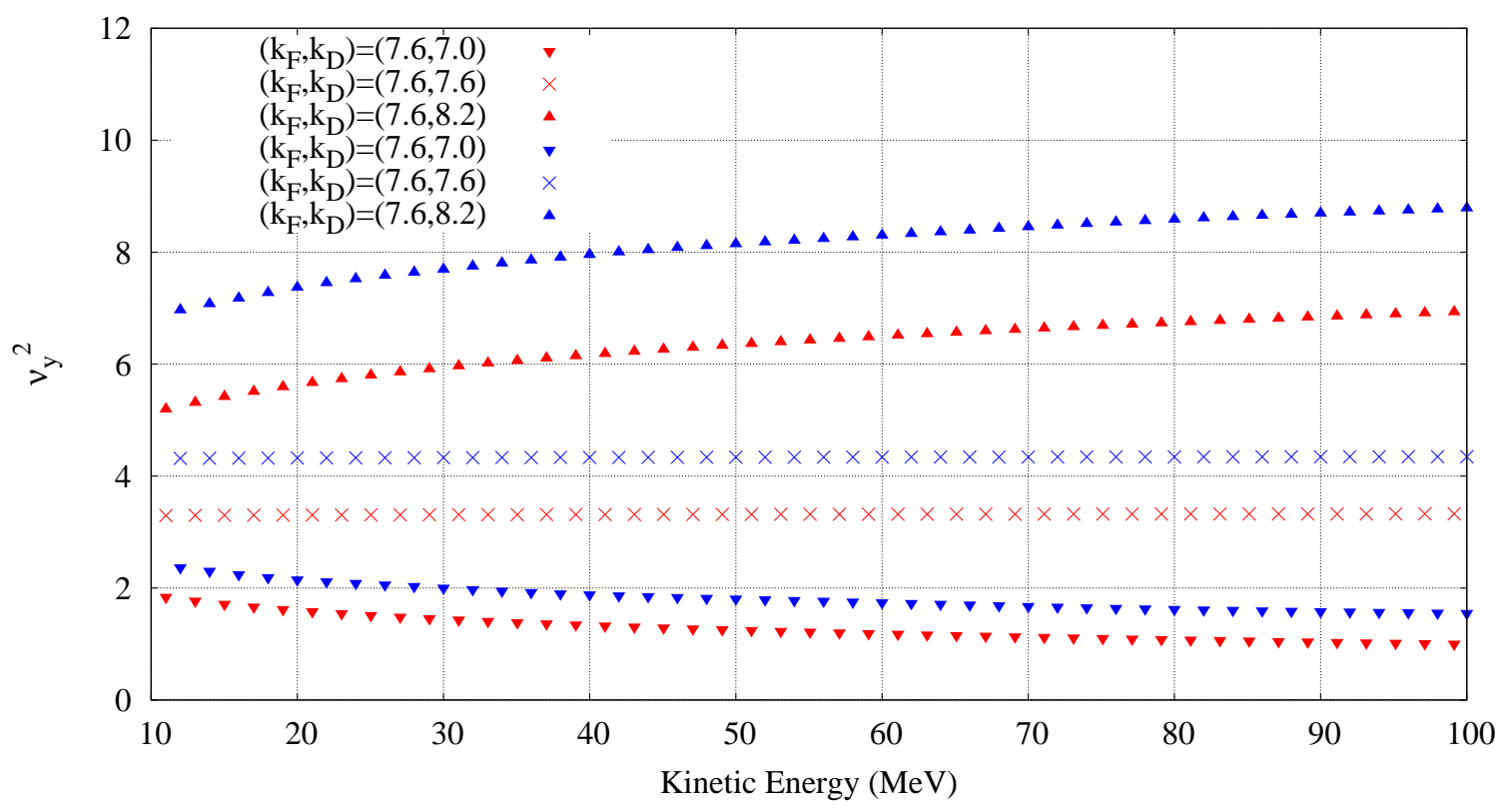

FIG. 34: Example of tune calculation using the tracking code ZGOUBI (blue) and comparison with the 1st order analytical formula 108 (red). To the first order in the method of averages, the differences are less than $20 \%$. The agreement should be better if the contribution of the second order term is taken into account (given that $g_{2 y}>0$ ).

into 121, one obtain:

$$
\ln \left(\frac{r_{2}}{r_{1}}\right)=\frac{1}{2(1+k)} \ln \left(\frac{\gamma_{2}^{2}-1}{\gamma_{1}^{2}-1}\right)=\frac{1}{2(1+k)} \ln \left(\frac{E_{2}^{2}-E_{m}^{2}}{E_{1}^{2}-E_{m}^{2}}\right)
$$

where $E_{i}$ refers to the total energy of the particle. We write: $E_{2}=E_{1}+\Delta E$ such that $\Delta E<<E_{1}$ (the energy gain per turn is in general negligible compared to the relativistic mass). Thus,

$$
\frac{E_{2}^{2}-E_{m}^{2}}{E_{1}^{2}-E_{m}^{2}}=\frac{\left(E_{1}+\Delta E\right)^{2}-E_{m}^{2}}{E_{1}^{2}-E_{m}^{2}} \approx 1+\frac{2 E_{1}}{E_{1}^{2}-E_{m}^{2}} \Delta E
$$

Injecting this into Eq. 123, and applying the exp, one obtains:

$$
\Delta r=r_{2}-r_{1}=r_{1}\left[\left(1+\frac{2 E_{1}}{E_{1}^{2}-E_{m}^{2}} \Delta E\right)^{\frac{1}{2(1+k)}}-1\right]
$$


or, in a more general form:

$$
\Delta r(E)=r(E)\left[\left(1+\frac{2 E}{E^{2}-E_{m}^{2}} \Delta E\right)^{\frac{1}{2(1+k)}}-1\right]
$$

where $\mathrm{r}$ is the radius of the closed orbit for which the total energy of the particle is E (can be obtained from Eq.120 above). Fig. 35 shows a comparison of the turn separation calculation using the tracking code ZGOUBI, with the analytical formula 126 .

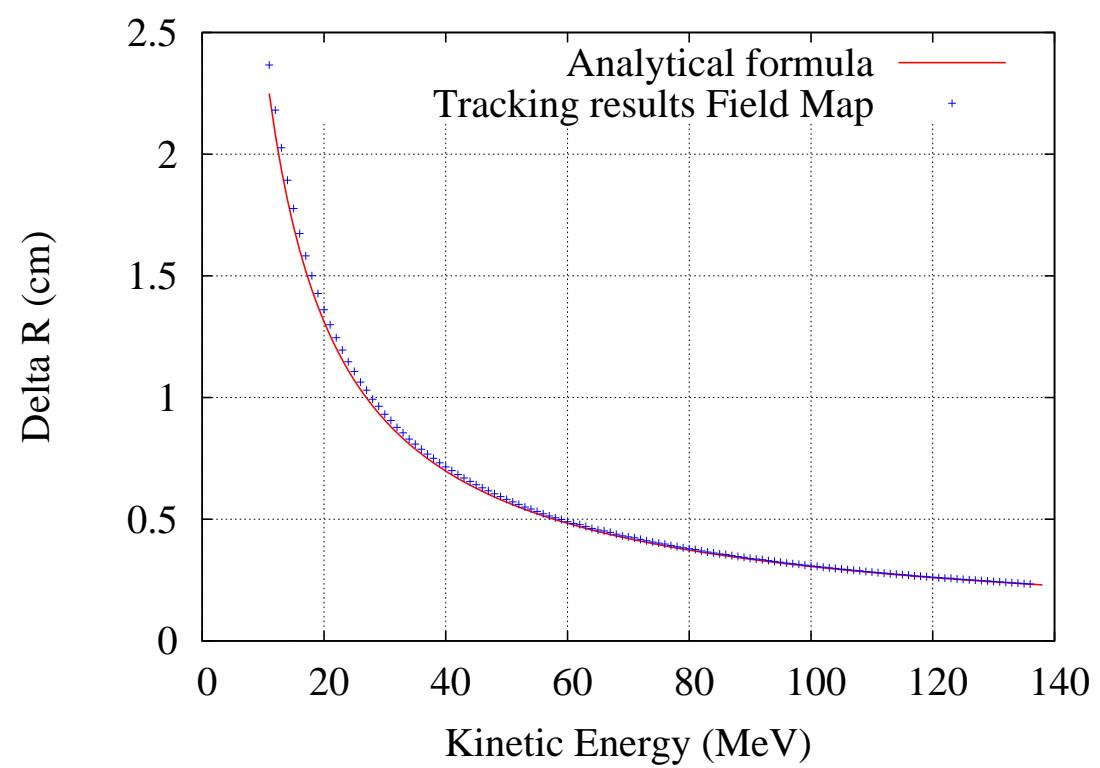

FIG. 35: Turn separation in the KURRI $150 \mathrm{MeV}$ FFAG as a function of the kinetic energy and comparison with the analytical formula (126).

[1] S. Lund, "Transverse equilibrium distribution", USPAS 2015, https://people.nscl.msu.edu/ lund/uspas/bpisc_2015/lec_set_02/tpd.pdf, p19-32.

[2] V.I. Arnold, "Mathematical Methods of Classical Mechanics", Second Edition, p. 129 (1981).

[3] J. M. Leinaas, "Classical Mechanics and Electrodynamic", University of Oslo (2009).

[4] Dieks D 2004 Space, time and coordinates in a rotating world Fundamental Theories in Physics vol 135 ed G Rizzi and M L Ruggiero (Dordrecht: Kluwer) pp 29-42

[5] D. L. Hetrick, "Dynamics of Nuclear Reactors", Chicago: University of Chicago Press (1971).

[6] S. L. Sheehy et al, "Progress on simulation of fixed field alternating gradient accelerators", MOPJE077, IPAC'15, Virginia, USA (2015).

[7] J. Scott Berg, "Correct Tracking in FFAGs", FFAG'05 workshop, Kyoto University (2005).

[8] K.R. Symon, D.W. Kerst, L.W. Jones, L.J. Laslett and K.M. Terwilliger, "Fixed-Field Alternating-Gradient Particle Accelerators", Phys. Rev.103, 1837 (1956).

[9] S. Machida, "Fixed Field Alternating Gradient", http://arxiv.org/pdf/1302.2026.pdf

[10] J.J. Livingood, "Principles of cyclic Particle Accelerators", D. Van Nostrand Co Ltd (1961).

[11] T. P. Wangler, "RF linear accelerators", WILEY-VCH Verlag GmbH \& Co.KGaA, Weinheim, pp.10-11 (2008).

[12] J. Dieudonné, "Calcul infinitésimal", Hermann, pp.419-422 (1968).

[13] https://www.bnl.gov/ffag14/

[14] S.L. Sheehy et al, "Characterization techniques for fixed field alternating gradient accelerators and beam studies using the 150 MeV proton FFAG", http://arxiv.org/pdf/1510.07459v2.pdf

[15] M. Reiser, "Theory and Design of Charged Particle Beams", Wiley Series in Beam Physics and Accelerator Technology, Wiley-VCH, Berlin (2008).

[16] M. Haj Tahar et al, "DESIGN OF THE INJECTION INTO THE $800 \mathrm{MeV}$ /amu HIGH POWER CYCLOTRON", MOPBA05, PAC'13, Pasadena,CA, USA (2013).

[17] N.N. Bogoliubov and Y.A. Mitropolskii, "Asymptotic methods in the theory of nonlinear oscillations", Gordon and Breach, New York (1961).

[18] A.A. Kolomensky and A.N. Lebedev, "THEORY OF CYCLIC ACCELERATORS", North-Holland publishing company, Amsterdam, pp 77-81 (1966).

[19] F. Méot, "Zgoubi Users Guide", Report CA/AP/470, BNL C-AD (2012)

[20] "The equations of motion in a non-inertial system differ from the equations in an inertial system by additional terms called inertial forces. This allows us to detect experimentally the non-inertial nature of a system. "[[]]

[21] The list of parameters in the following notation is summarized in the nomenclature. 
[22] A commonly used relation giving the maximum deviation around the closed orbit is: $x_{m}^{2}=\epsilon \beta_{m} / \pi$ where $\beta_{m}$ is the maximum value of the periodic betatron function.

[23] From now on, unless otherwise specified, the magnetic field B refers only to the vertical component of the field.

[24] This result is a generalization to what can be found in the Symon paper [8]. We introduce the radial dependence of the tune. In other words, no assumption is made regarding the constancy of the tune or the similarity of the orbits. Also, we distinguish between the $\mathrm{F}$ and $\mathrm{D}$ magnets, which is equivalent to introducing an azimuthal variation of the scaling factors.

[25] Only externally applied forces are considered here, i.e no collective effects such as the space charge effects.

[26] Another stability island exists for larger $\mathrm{k}$ values, but we restrict our analysis to a KURRI type FFAG for which the design value of $\mathrm{k} \approx 7.6$ 